\title{
Piloted Simulator Evaluation of Maneuvering Envelope Information for Situational Awareness
}

\author{
Thomas Lombaerts* \\ German Aerospace Center DLR, \\ 82234 Weßling, Germany \\ Stefan Schuet ${ }^{\dagger}$ \\ NASA Ames Research Center, \\ Moffett Field, CA 94035, USA \\ Diana Acosta ${ }^{\ddagger}$ and John Kaneshige ${ }^{\S}$ \\ NASA Ames Research Center, Moffett Field, CA 94035, USA \\ Kimberlee Shish \\ Millennium Engineering 83 Integration Company, NASA Ames Research Center, \\ Moffett Field, CA 94035, USA \\ Lynne Martin" \\ San Jose State University, NASA Ames Research Center, Moffett Field, CA 94035, USA
}

\begin{abstract}
The implementation and evaluation of an efficient method for estimating safe aircraft maneuvering envelopes are discussed. A Bayesian approach is used to produce a deterministic algorithm for estimating aerodynamic system parameters from existing noisy sensor measurements, which are then used to estimate the trim envelope through efficient highfidelity model-based computations of attainable equilibrium sets. The safe maneuverability limitations are extended beyond the trim envelope through a robust reachability analysis derived from an optimal control formulation. The trim and maneuvering envelope limits are then conveyed to pilots through three axes on the primary flight display. To evaluate the new display features, commercial airline crews flew multiple challenging approach and landing scenarios in the full motion Advanced Concepts Flight Simulator at NASA Ames Research Center, as part of a larger research initiative to investigate the impact on the energy state awareness of the crew. Results show that the additional display features have the potential to significantly improve situational awareness of the flight crew.
\end{abstract}

\section{Introduction}

$\mathrm{T}^{\mathrm{N}}$ all transportation systems, but especially in civil aviation, safety is of paramount importance. Many 1 developments focus on improving safety levels and reducing the risks of life threatening failures. In a recent study by the Commercial Aviation Safety Team (CAST) and the International Civil Aviation Organization (ICAO), it can be observed that loss of control in flight (LOC-I) is the most frequent primary accident

\footnotetext{
${ }^{*}$ Researcher at DLR, Institute of System Dynamics and Control, Department of Aircraft Dynamics, e-mail: thomas.lombaerts@dlr.de, currently Marie Curie Fellow and visiting researcher at NASA Ames Research Center, Intelligent Systems Division, Adaptive Control and Evolvable Systems (ACES) Group, e-mail: thomas.lombaerts@nasa.gov, AIAA Member.

${ }^{\dagger}$ Researcher at NASA Ames Research Center, Intelligent Systems Division, Mail Stop 269-1, AIAA Member.

${ }_{\ddagger}^{\ddagger}$ Aerospace Engineer at NASA Ames Research Center, Intelligent Systems Division, Mail Stop 269-1, AIAA Member.

$\S$ Computer Engineer at NASA Ames Research Center, Intelligent Systems Division, Mail Stop 269-1, AIAA Senior Member.

IResearcher at NASA Ames Research Center, Intelligent Systems Division, Mail Stop 269-1, AIAA Member.

" Researcher at NASA Ames Research Center, Human Systems Integration Division, Mail Stop 262-4.
} 
cause ${ }^{1}$ LOC-I accidents have a variety of contributing factors, occurring individually or in combination, such as a system malfunction, atmospheric disturbances (e.g. turbulence or icing), and loss of situational awareness by the crew. An important aspect during operations under system malfunction or atmospheric disturbance is that the crew needs to maintain awareness through possibly time changing degradation of aircraft performance characteristics. The CAST established a specialized international safety analysis team to study the apparent growing trend in loss of Airplane State Awareness (ASA) by the flight crew. This team has produced recommended safety enhancements that include the research, development, and implementation of technologies for enhancing flight crew awareness of airplane energy state (SE 207) ${ }^{[2}$ The research presented in this paper provides a means to address these recommendations by interfacing on-board safe flight envelope estimation capability with cockpit displays and testing them on commercial airline crews tasked with flying challenging approach and landing scenarios.

In full generality, the safe flight envelope is an entire set of safe aircraft state and control input combinations. Because of the underlying nonlinear aircraft dynamics, it is challenging to accurately map this set fast enough to provide the pilot or automation system with reliable information that reflects diverse and rapidly changing aircraft operational conditions. A variety of methods for tackling this challenge have been investigated in previous studies covering wind tunnel testing, flight test experiments, and high-fidelity model-based computation ${ }^{3] 4}[\sqrt[5]{6}$ The approach proposed in this paper, builds on previous work, where the trim envelope is estimated through efficient high-fidelity model-based computations of attainable equilibrium sets, which in turn depend on aerodynamic coefficient identification from air data, inertial, and GPS measurements. The safe maneuverability limitations are then extended beyond the trim envelope through a robust reachability analysis derived from an optimal control formulation that leverages the principle of time scale separation. The theoretical details covering this approach in-depth are available in previous publications.$^{7[8] 9}$

This paper is focused on applying the previously developed theory to the practice of conveying meaningful aircraft state awareness information to the crew. This is accomplished by translating the trim and maneuvering envelope boundaries to indicators for three axes on the primary flight display, namely for airspeed, vertical speed, and bank angle, in a manner that leverages existing conventions already familiar to commercial pilots. The impact on the energy state awareness of the crew was then evaluated in the full motion Advanced Concepts Flight Simulator at NASA Ames Research Center. Commercial airline crews flew multiple problematic approach and landing scenarios simulating the airspace around Memphis International Airport.

The structure of this paper is as follows. In Sec. III the method for estimating the trim and maneuvering envelopes is summerized from previous research. The presentation of information to the crew is elaborated in Sec. III] The experiment method is described in Sec. IV] Sec. V] discusses the results. Conclusions and recommendations can be found in Sec. VI

\section{Estimation of the envelope boundaries}

The safe maneuvering envelope is a fundamental property of the aircrafts design and overall current state of health. By definition it determines the overall capability of the aircraft. If actively monitored in time, it may function as an early warning system as well as provide anticipatory guidance to help avoid loss of control. Improved methods for tracking aircraft maneuverability in real-time may therefore effectively help pilots avoid inappropriate crew response and further prevent or recover aircraft from upset conditions.

This section summarizes in some detail our previous work on developing computationally efficient methods for on-board envelope estimation from real-time aircraft sensor data. This capability provides the underlying information required in Sec. III for constructing new primary flight display features.

\section{A. Mathematical model postulation and parameter identification}

The aircraft model used for the simulator study discussed later in this paper, is the Advanced Concepts Transport (ACT) model, which is representative of a mid-size, two-engine, jet transport aircraft as shown in Fig. 1(a) It has the general characteristics of a wide-body, T-tail, low wing airplane with twin turbofan 
engines located under the wings, and physical dimensions similar to those of a Boeing 757 aircraft 10 The full simulation model is based on a Lockheed-Georgia Company commercial transport concept developed in 1983, which was designed as a platform for testing advanced aircraft simulator concepts with active flight controls, representative of modern fly-by-wire control systems.

Given this high fidelity simulation model, the goal in this subsection is to develop a reduced complexity model that allows one to study particular characteristics of the higher fidelity model in a computationally efficient manner. For the application of interest, this is best accomplished by using the principle of time scale separation to find a mathematical description of the flight dynamics focused on the variables that drive the slow dynamics of the aircraft state. The basic concept is depicted in Fig. 1(b), which illustrates that the nine dimensional nonlinear problem, relating thrust and control surface deflection to the angular rates and ultimately the velocity vector in polar coordinate form, is decomposed into three coupled mappings between high, middle, and low bandwidth variables.

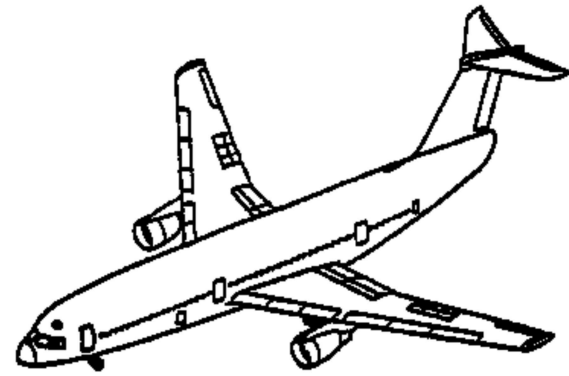

(a) ACT Model

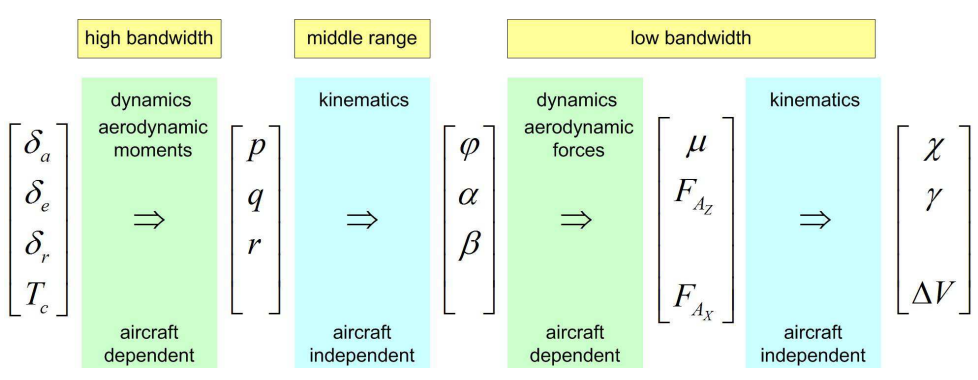

(b) Separation of dynamics into high bandwidth, middle range, and low bandwidth variables

Figure 1.

The reduced complexity model is developed from the physical relationships between the low bandwidth variables, where net engine thrust $T$, angle of attack $\alpha$, side-slip angle $\beta$, and roll angle $\phi$ are treated as known virtual inputs. The aircraft state vector of interest is then comprised of the total air speed $V$, and flight path angle $\gamma$. After making use of the small angle assumption for $\alpha$ and $\beta$ one obtains the following expression for the aircraft state dynamics,

$$
\left[\begin{array}{c}
\dot{V} \\
\dot{\gamma}
\end{array}\right] \approx\left[\begin{array}{c}
-\frac{\rho S}{2 m} V^{2} \tilde{C}_{D_{0}}-g \sin \gamma \\
-\frac{g}{V} \cos \gamma
\end{array}\right]+\left[\begin{array}{l}
1 \\
0
\end{array}\right] \frac{T}{m}+\left[\begin{array}{c}
-\frac{\rho S}{2 m} V^{2}\left(\tilde{C}_{D_{\alpha}} \alpha+C_{D_{\alpha^{2}}} \alpha^{2}\right) \\
\frac{\rho S}{2 m} V\left(\tilde{C}_{L_{0}}+C_{L_{\alpha}} \alpha\right) \cos \phi
\end{array}\right]+\left[\begin{array}{c}
0 \\
-\frac{\rho S}{2 m} V C_{Y_{\beta}} \beta \sin \phi
\end{array}\right],
$$

where $g$ is the acceleration due to gravity, $m$ is the mass of the aircraft, $S$ is the net wing surface area, and $\rho$ is the air density. For the particular simulation discussed in this paper, the following fixed constants were assigned: $m=90719 \mathrm{~kg}, g=9.802 \mathrm{~m} / \mathrm{s}^{2}$, and $S=181.25 \mathrm{~m}^{2}$. The altitude dependent air density $\rho$ was assumed known according to the U.S. Standard Atmosphere published in Ref. 11, Furthermore, within this setup, the aerodynamic coefficients take into account the influences of the secondary control surface deflections:

$$
\begin{aligned}
\tilde{C}_{D_{0}} & =C_{D_{0}}+C_{D_{\delta_{\mathrm{a}}}} \delta_{\mathrm{a}}+C_{D_{\delta_{\mathrm{sp}}}} \delta_{\mathrm{sp}}+C_{D_{\delta_{\mathrm{fl}}}} \delta_{\mathrm{fl}}+C_{D_{\delta_{\mathrm{lg}}}} \delta_{\mathrm{lg}} \\
\tilde{C}_{D_{\alpha}} & =C_{D_{\alpha}}+C_{D_{\alpha \delta_{\mathrm{fl}}}} \delta_{\mathrm{fl}} \\
\tilde{C}_{L_{0}} & =C_{L_{0}}+C_{L_{\delta_{\mathrm{a}}}} \delta_{\mathrm{a}}+C_{L_{\delta_{\mathrm{sp}}}} \delta_{\mathrm{sp}}+C_{L_{\delta_{\mathrm{fl}}}} \delta_{\mathrm{fl}}+C_{L_{\delta_{\mathrm{lg}}}} \delta_{\mathrm{lg}},
\end{aligned}
$$

where the deflections due to ailerons, spoilers, flaps, and landing gear are provided by $\delta_{\mathrm{a}}, \delta_{\mathrm{sp}}, \delta_{\mathrm{ff}}$, and $\delta_{\mathrm{lg}}$, respectively. The influence of the ailerons on lift and drag are typically negligible for conventional aircraft configurations. However, for the ACT design, we found that aileron deflection had a significant impact on lift and drag. For that reason, terms to cover aileron effects were included in the model equations above. 
An important practical aspect of this reduced complexity model is that it is affine with respect to the aerodynamic coefficients.

In general, models for the pitching moment coefficient are aircraft type dependent. For the ACT simulator model in particular, the model expression was developed through a standard model structure selection analysis. ${ }^{[12] 13}$ The resulting expression was

$$
\begin{aligned}
C_{m}= & C_{m_{0}}+C_{m_{\alpha}} \alpha+C_{m_{\alpha^{2}}} \alpha^{2}+C_{m_{q} \bar{q}} \frac{q \bar{c}}{V}+C_{m_{\delta_{\mathrm{e}}}} \delta_{\mathrm{e}}+C_{m_{\delta_{\mathrm{e}}^{2}}} \delta_{\mathrm{e}}^{2}+C_{m_{i_{\mathrm{h}}}} i_{\mathrm{h}}+C_{m_{T}} \frac{T}{\bar{q} d_{\mathrm{eng}}^{2}}+ \\
& +C_{m_{\delta_{\mathrm{a}}}} \delta_{\mathrm{a}}+C_{m_{\delta_{\mathrm{sp}}}} \delta_{\mathrm{sp}}+C_{m_{\delta_{\mathrm{fl}}}} \delta_{\mathrm{fl}}+C_{m_{\delta_{\mathrm{lg}}}} \delta_{\mathrm{lg}},
\end{aligned}
$$

where $\bar{c}$ is the mean aerodynamic chord, $q$ is the current pitch rate, $\delta_{\mathrm{e}}$ is the elevator deflection, and $i_{\mathrm{h}}$ is the stabilizer deflection. A more detailed derivation of Eq. (11) and Eq. (2) is available in Ref. 14, [TODO: check EuroGNC paper citation]

\section{B. Aerodynamic Parameter identification}

Ultimately, we want to find a way to use Eq. (11) and Eq. (2) to numerically characterize the safe flight envelope for a particular aircraft. This task however, first requires the aircraft type and flight condition dependent aerodynamic coefficients that constitute the vectors

$$
\mathbf{c}=\left(C_{D_{0}}, C_{D_{\delta_{\mathrm{a}}}}, C_{D_{\delta_{\mathrm{sp}}}}, C_{D_{\delta_{\mathrm{fl}}}}, C_{D_{\delta_{\mathrm{Ig}}}}, C_{D_{\alpha}}, C_{D_{\alpha \delta_{\mathrm{fl}}}}, C_{L_{0}}, C_{L_{\alpha}}, C_{L_{\delta_{\mathrm{a}}}}, C_{L_{\delta_{\mathrm{sp}}}}, C_{L_{\delta_{\mathrm{fl}}}}, C_{L_{\delta_{\mathrm{lg}}}}\right)
$$

and

$$
\mathbf{m}=\left(C_{m_{0}}, C_{m_{\alpha}}, C_{m_{\alpha^{2}}}, C_{m_{\underline{q} \bar{v}}}, C_{m_{\delta_{\mathrm{e}}}}, C_{m_{\delta_{\mathrm{e}}^{2}}}, C_{m_{i_{\mathrm{h}}}}, C_{m_{T}}, C_{m_{\delta_{\mathrm{a}}}}, C_{m_{\delta_{\mathrm{sp}}}}, C_{m_{\delta_{\mathrm{fl}}}}, C_{m_{\delta_{\mathrm{lg}}}}\right) .
$$

The estimation of these vectors is accomplished through a system identification step that essentially fits the above described dynamics model to any particular aircraft, or higher fidelity simulation model, such as the ACT model.

\section{Sensor Information}

An inertial sensor package, consisting of rate gyros and accelerometers, provides angular rates and accelerations as well as specific forces. It is assumed that a state estimation algorithm is available, taking into account sensor disturbances (biases and/or noise), and compensating for them.15

\section{Bayesian Inference Approach}

A specialized Bayesian probabilistic approach was developed to infer the unknown aerodynamic coefficients from the noisy sensor measurements and to quantify the estimation uncertainty. With this approach the forward model provided by equations Eq. (11) and Eq. (2) is used to define a noisy sensor measurement process, which then enables one to derive the posterior pdf

$$
p(\mathbf{c}, \mathbf{S} \mid \text { Observed Data })
$$

that specifies the probability density of the unknown parameter values $\mathbf{c}$ and inverse measurement noise covariance matrix $\mathbf{S}$, given the relevant history of observed data and prior information. With the modeling and additive noise assumptions used in the derivation, the posterior pdf Eq. (3) is log-concave in either $\mathbf{c}$ or $\mathbf{S}$ when the other variable is held fixed. This enables the efficient computation of a local optimal estimate using a deterministic block coordinate descent method to break the solution process into steps that require solving simple convex optimization problems. Furthermore, the coefficient parameter estimation uncertainty and Bayesian model evidence can also be estimated using closed form expressions. Significantly, more information about the identification procedure is available in Ref. 9 , 


\section{Estimation of the trim envelope}

One important feature of the reduced complexity model Eq. (1) is that it enables a rapid numerical analysis of the set of trimmable aircraft states,

$$
\{\mathbf{x} \mid \mathbf{f}(\mathbf{x}, \mathbf{u} ; \mathbf{c})=0,(\mathbf{x}, \mathbf{u}) \in \mathcal{B}\},
$$

where $\mathbf{f}(\mathbf{x}, \mathbf{u} ; \mathbf{c})$ implements the right hand side of Eq. (1) with state vector $\mathbf{x}=(V, \gamma)$, and input vector $\mathbf{u}=\left(T, \alpha, \beta, \phi, \delta_{\mathrm{a}}, \delta_{\mathrm{sp}}, \delta_{\mathrm{fl}}, \delta_{\mathrm{lg}}\right)$. The set $\mathcal{B}$ contains all allowable states and virtual inputs. The trim envelope defined by Eq. (44) is important because it represents an a-priori safe flight envelope.

With the reduced complexity model, and a given aerodynamic parameter vector c, characterizing the set of trimmable points then involves setting the top and bottom equations on the right hand side of (1) equal to zero. The bottom equation is solved for angle of attack $\alpha$ in terms of the other variables in that equation, which do not include thrust $T$. The top equation is then solved for $T$ into which we substitute the previous solution for $\alpha$. The result is a closed form solution for the required thrust and alpha needed to achieve trim for any given state vector $\mathbf{x}$ and fixed values for the other virtual inputs $\beta$ and $\phi$, as well as control surface deflections $\delta_{\mathrm{a}}, \delta_{\mathrm{sp}}, \delta_{\mathrm{fl}}$, and $\delta_{\mathrm{lg}}$. If it turns out that the required $(T, \alpha)$ pair falls within $\mathcal{B}$, then $\mathbf{x}$ is a trimmable state. In all, this process enables one to code a computationally efficient indicator function

$$
\mathcal{I}(\mathbf{x})= \begin{cases}1 & \text { if } \mathbf{x} \in \text { Trim Envelope } \\ 0 & \text { otherwise. }\end{cases}
$$

The trim envelope is then revealed by using $\mathcal{I}(x)$ to filter any grid of test aircraft state vectors down to those for which a trim condition is achievable within minimum and maximum constraints on $\mathbf{x}$ and $\mathbf{u}$. In addition, one should check whether the achieved trim points are stable. This involves checking the eigenvalues associated with the local linear approximation to Eq. (1) at each trim point, and as before, a closed form expression can be found.

For fixed aircraft configuration, roll angle, and side-slip angle, the virtual inputs are thrust and angle of attack. Figure 2(a) illustrates how the constraints on these inputs determine the envelope boundaries. The minimum speed boundary corresponds to the maximum angle of attack at which stall occurs. The maximum speed boundary is determined either by the minimum permissible angle of attack, or by the maximum sustainable structural load, whichever requires the slower speed. The upper and lower flight path angle $\gamma$ boundaries are defined by the maximum and minimum net excess thrust over drag, respectively. The upper flight path angle boundary, which defines $\gamma_{\max }$, would have a similar shape as the lower boundary for $\gamma_{\min }$, but a set of unstable trim points has been left out of the envelope at the upper boundary, which makes it distinctly different in shape than the lower boundary, and which further makes the back side of the power curve effect less apparent. 9

\section{Estimation of the maneuvering envelope}

Even under normal operations, aircraft can and do safely exit and return to the trim envelope, especially during the descent phase of flight. Therefore, the preferred definition of the maneuvering envelope should be the set of states that the aircraft can safely attain, and from which it can recover back to trim. Finding this set, requires solving for the forward and backward reachable sets. The forward reachable set contains the states that can be reached from at least one point in the trim envelope. The backward reachable set contains the initial states from which one can maneuver the aircraft back to the trim envelope. The safe maneuvering flight envelope is then defined as the intersection of these forward and backward reachable sets. This interpretation is illustrated in Fig. 2(b), In addition to its contribution to the safe maneuvering envelope, the backward reachable set is also considered a survivable flight envelope. After an upset due to damage, turbulence, wake encounter etc., it is possible to bring the aircraft back to a safe trim condition as long as the current flight condition is situated inside the backwards reachable set.

Previous work has rigorously shown that reachability based maneuvering envelopes can be calculated by first reformulating the problem within an optimal control framework, $\frac{16}{2}$ and then using level set methods to 
complete the calculation $]^{7 / 8}[17$ In addition, robustness with respect to uncertainty in the identified model is readily incorporated into the solution process.

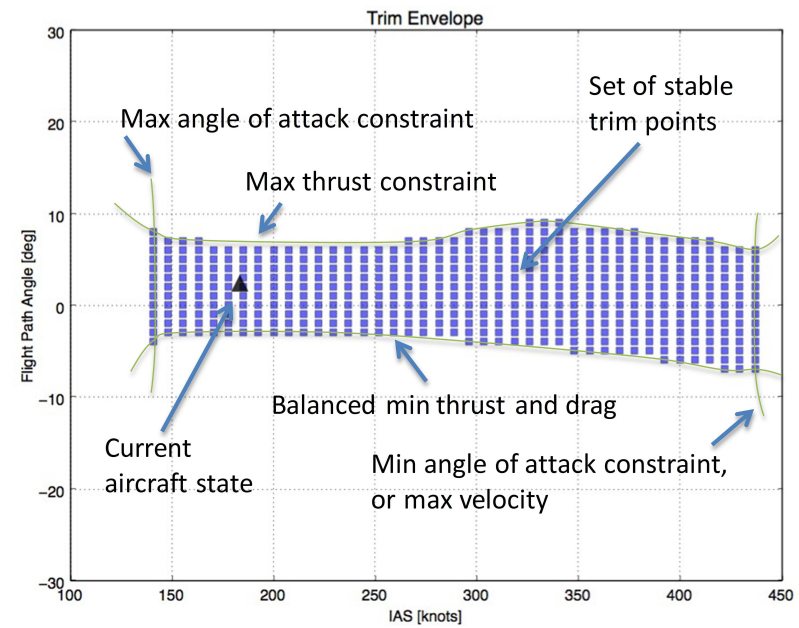

(a) Trim Envelope

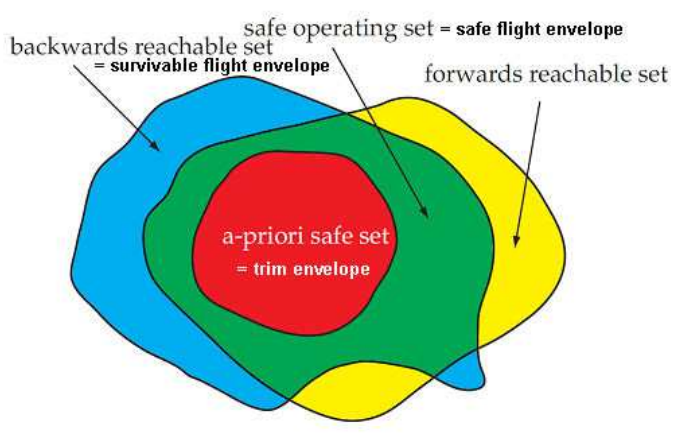

(b) Maneuvering Envelope

Figure 2. (a) $(V, \gamma)$ trim envelope for ACT model at 15,000 ft. (b) Safe maneuvering envelope as intersection between forwards and backwards reachability, modified from source: van Oort 18

\section{E. Example trim and maneuvering envelopes}

The basic affects of altitude and aircraft configuration on the trim and maneuvering envelopes are shown in Fig. 3. Figure 3(a) shows the envelope for the clean aircraft configuration at 15,000 ft. In Fig. 3(b) the altitude is increased to 30,000 ft, which causes the envelope to shrink because the reduced air density at higher altitude decreases lift, drag, and maximum engine thrust authority. Flap deployment increases the lift, drag, and maximum angle of attack while also requiring slower maximum airspeed due to structural limitations with flap extension. The resulting combination of these effects, which enable the aircraft to safely sustain slower airspeed, is shown for full flap deployment in Fig. 33(c). Adding gear and spoilers further increases drag, and by comparing 3(c) to 3(d) one observes that greater descent angles are possible at higher airspeed.

\section{F. Pitching moment control authority for a fixed stabilizer position}

Depending on the stabilizer position and elevator authority, there may be stricter upper limits on speed and climb rate combinations than those produced by the limits on $\alpha$ and $T$ discussed above. This important information can be incorporated into the trim envelope by adding consideration of the moment equation Eq. (2). At any particular trim condition one can assume $\alpha, T, i_{\mathrm{h}}, \delta_{\mathrm{a}}, \delta_{\mathrm{sp}}, \delta_{\mathrm{fl}}$, and $\delta_{\mathrm{lg}}$ are fixed and $q=0$. By setting the left hand side of Eq. (2) to zero one observes that a specific elevator input $\delta_{\mathrm{e}}$ is then required to balance the equation. If the required $\delta_{\mathrm{e}}$ falls within the elevator control envelope, then the trim point remains a viable option; otherwise, the point must be removed from the envelope.

For small to moderate stabilizer positions the elevator saturation limits have no impact on the shape of the trim envelope. However, for large stabilizer deflection angles the effect can be significant as shown in Fig. 4. With the stabilizer position set to $0^{\circ}$, Fig. 4(a) shows that the elevator deflections required to balance the pitching moment vary between $-8^{\circ}$ and $15^{\circ}$ for the entire trim envelope. This requirement falls well within the elevator authority from $-30^{\circ}$ to $+20^{\circ}$, so there is no issue in this case. However, Fig. 4(b) and $4(\mathrm{c})$ show the situation that occurs when the stabilizer is fixed at $6^{\circ}$ and $8^{\circ}$ nose down, respectively. The pitch up moment generated by these stabilizer positions requires elevator deflections beyond the maximum 


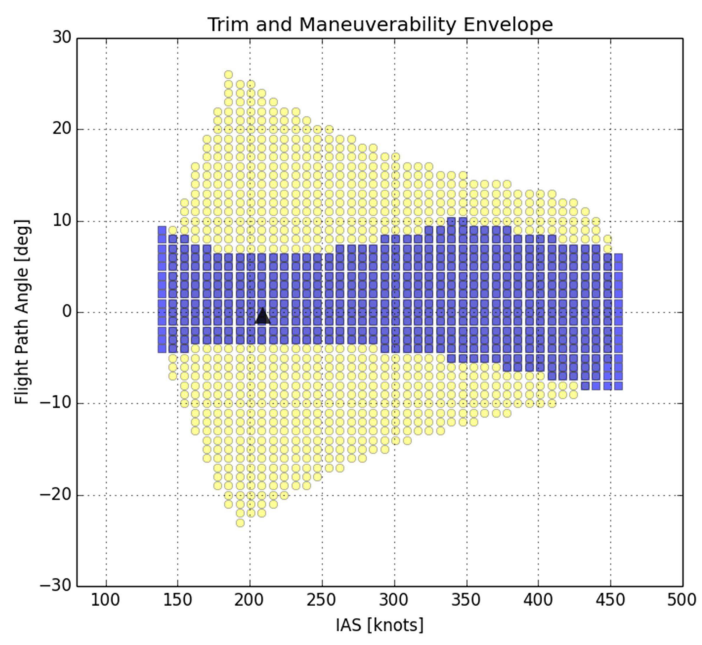

(a) nominal at $15,000 \mathrm{ft}$

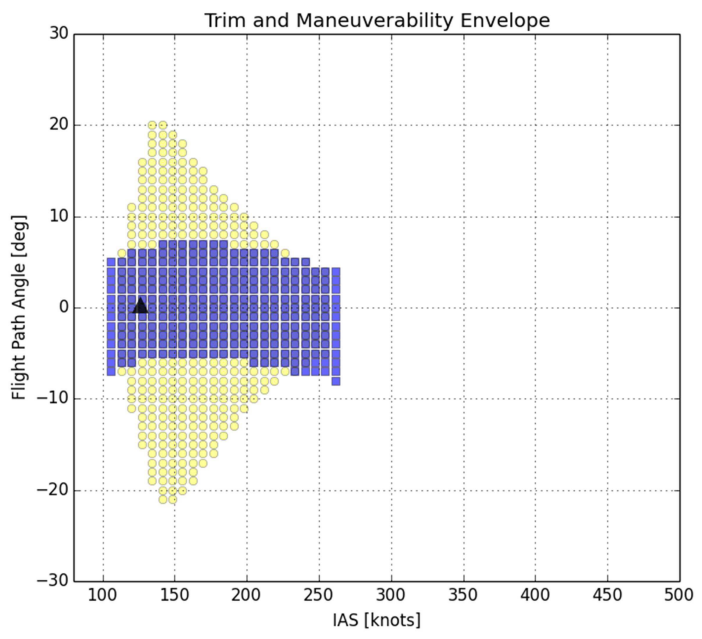

(c) full flaps at $15,000 \mathrm{ft}$

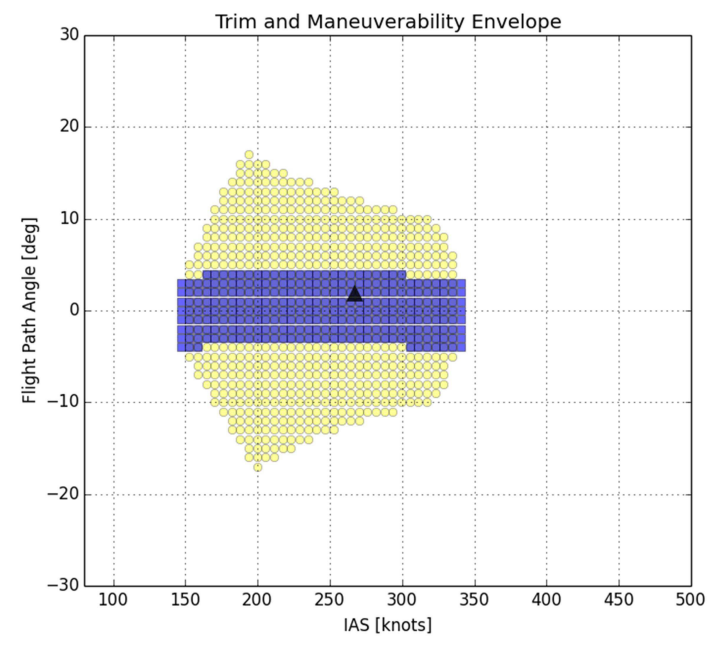

(b) nominal at $30,000 \mathrm{ft}$

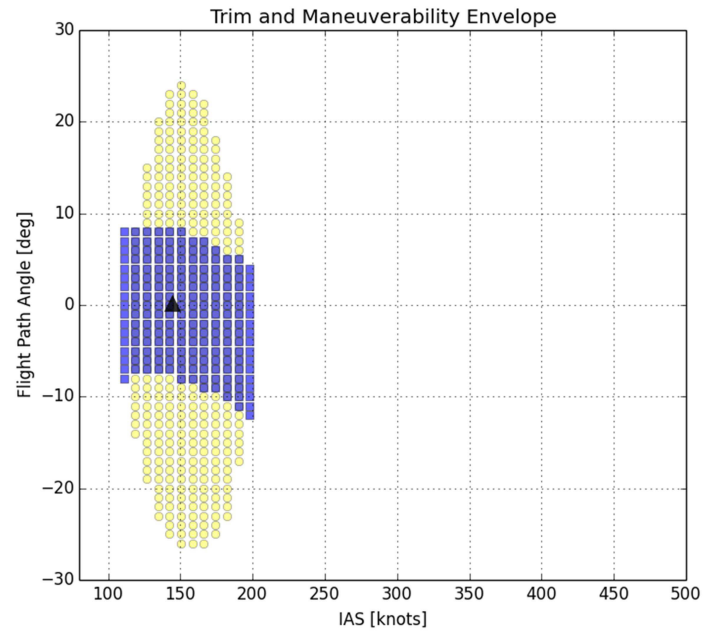

(d) full flaps, gear and spoilers at 13,000 ft

Figure 3. $\quad V, \gamma$ trim (blue) and maneuvering (yellow) envelopes of ACT model in differenct configurations for bank angle $\phi=0$ and sideslip angle $\beta=0.10 \%$ uncertainty included for the maneuvering envelope. The black triangle corresponds to the current flight condition of the aircraft during the simulation. 
limit at $\delta_{\mathrm{e}_{\max }}=20^{\circ}$, and this additional input constraint significantly decreases the size of the trim envelope. For level flight $\gamma=0^{\circ}$, the conventional maximum airspeed of $V=180 \mathrm{~m} / \mathrm{s}$ is reduced to $V=140 \mathrm{~m} / \mathrm{s}$ for $i_{\mathrm{h}}=-6^{\circ}$, and $V=100 \mathrm{~m} / \mathrm{s}$ for $i_{\mathrm{h}}=-8^{\circ}$. This is important information that may significantly increases crew situational awareness.

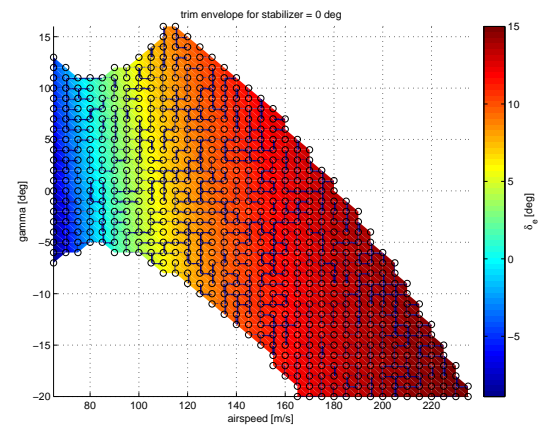

(a) $i_{\mathrm{h}}=0^{\circ}$

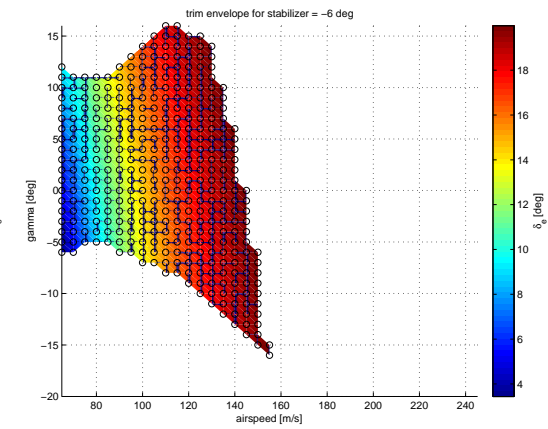

(b) $i_{\mathrm{h}}=-6^{\circ}$

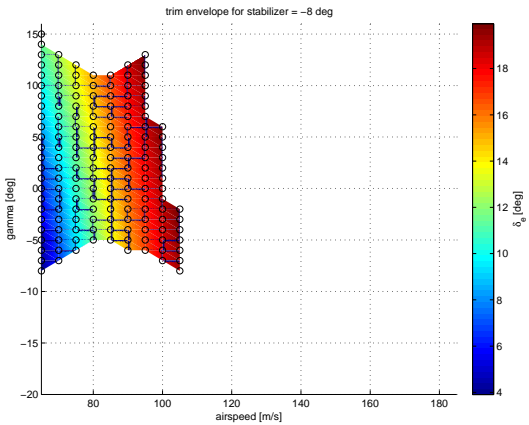

(c) $i_{\mathrm{h}}=-8^{\circ}$

Figure 4. $\quad V, \gamma$ trim envelopes of the ACT model for different stabilizer positions, with bank angle $\phi=0$ and sideslip angle $\beta=0$. Elevator saturation limits are $-30^{\circ}$ and $+20^{\circ}$.

\section{G. Maximum bank angle calculation}

The maximum bank angle $\phi$ is calculated by finding the angle at which the remaining vertical component of the maximum achievable lift vector (with respect to $\alpha$ ) is still able to balance the weight of the aircraft. This means that banking the aircraft at the current airspeed beyond this maximum angle will result in a stall. The concept is illustrated in Fig. [5, and the corresponding calculation is

$$
\pm \phi_{\max }= \pm \min \left\{\arccos \left(\frac{W}{C_{L_{\max }} 1 / 2 \rho V^{2} S \cos \gamma}\right), 35^{\circ}\right\}
$$

where $C_{L_{\max }}=C_{L_{0}}+C_{L_{\alpha}} \alpha_{\max }$. In this calculation, the current values for airspeed $V$ and flight path angle $\gamma$ are used, along with the up-to-date values for $C_{L_{0}}$ and $C_{L_{\alpha}}$ provided by the identification algorithm. The maximum angle of attack $\alpha_{\max }$ is further assumed known, but in principle it too could be updated from existing sensor data. Finally, Eq. (6) also reflects the fact that for a conventional civil airliner, the maximum bank angle does not typically exceed $35^{\circ}$.

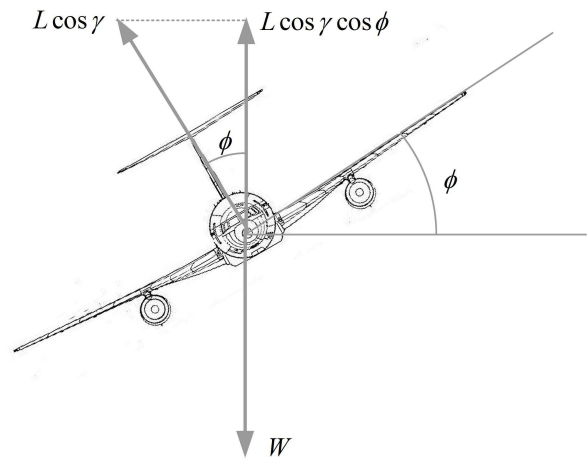

Figure 5. Force balance between lift and weight in a turn 
The critical aspect of the calculation is that the bank angle limits $\pm \phi_{\max }$ become increasingly restricted as the aircraft slows to the stall airspeed - and at stall speed, no bank authority remains. In an icing scenario, one expects the maximum lift capability of the aircraft, established by the value of $C_{L_{\max }}$, to decrease, while the total aircraft weight $W$ remains the same 3 As a consequence, $\cos \phi_{\max }$ increases, which results in a decrease of $\phi_{\max }$. Some typical values for the ACT model without icing are shown in Table 1]

\begin{tabular}{ccc}
\hline IAS $[\mathrm{kts}]$ & $\alpha\left[^{\circ}\right]$ & $\phi_{\max }\left[^{\circ}\right]$ \\
\hline 222 & 4 & \pm 70 \\
161 & 9 & \pm 45 \\
142 & 15 & $\approx 0$ \\
\hline
\end{tabular}

Table 1. A few typical values for the maximum bank angle at different airspeeds, computed for the ACT model.

\section{H. Computational efficiency}

The above methods for system identification, trim envelope estimation, and maneuvering envelope estimation are computationally efficient enough to dynamically re-recompute as aircraft conditions change, or to compute extended envelopes by sweeping over values for $\phi$ and $\beta$. For example, with the resolution setting used to generate the envelopes in Fig. 3, the system identification step took about $20 \mathrm{~ms}$, the trim envelope estimation step took $80 \mathrm{~ms}$, and the maneuvering envelope estimation step took about $2 \mathrm{~s}$. The additional computational cost required for the pitching moment control and bank angle limit calculations is negligible. The calculations were performed on a 2.6 GHz MacBook Pro laptop computer.

\section{Communicating the envelope information to the crew}

The envelope information derived in the previous section may be useful to the control system, the automation system, and to the crew. In this section one approach designed to enhance crew awareness through existing displays and symbology is discussed. Subsection A cover the conversion of the trim and maneuvering envelope information into airspeed and vertical speed markers for use on the primary flight display (PFD). Sections $\mathrm{B}$ and $\mathrm{C}$ then discuss how the maximum pitch and bank angle information are presented on the PFD, respectively.

\section{A. Mapping envelope information to PFD limit indicators}

Due to the nonlinear nature of the underlying dynamics model, the calculated trim and maneuvering envelopes are defined by two-dimensional non-convex sets that inherently contain more information than can be represented by one-dimensional limit indicators on the PFD. Nonetheless, the PFD is the essential tool for effectively conveying the most important envelope information to the pilot.

A straightforward approach is to generate PFD indicators based on the current aircraft state and configuration (flaps, gear, speed brakes, and stabilizer position). The goal, then, is to display the minimum and maximum safe indicated airspeeds at the current flight path angle, as well as the minimum and maximum steady-state (or maneuvering) vertical speeds at the current airspeed. The situation is depicted in Fig. 6. With the current aircraft state at $\mathrm{A}$, corresponding to a particular $\left(V_{\text {crnt }}, \gamma_{\text {crnt }}\right)$ pair, the minimum and maximum airspeeds are obtained from the subset of estimated trim points with $\gamma=\gamma_{\text {crnt }}$, while the minimum and maximum steady state flight path angles are found from the subset of trim points with $V=V_{\text {crnt }}$. An analogous process is used to find the remaining extremal points for the maneuvering envelope as shown in the figure. Of course, the true airspeed (TAS) envelope boundaries are first converted to indicated airspeed (IAS) by accounting for air density before they are displayed on the airspeed tape on the left side of the

\footnotetext{
${ }^{a}$ In some icing scenarios it is possible for the weight of the aircraft to increase with ice accretion. The incorporation of this aspect requires further online estimation of the aircraft weight, which is not a topic explicitly covered in this paper.
} 
PFD. Likewise, the flight path angle $\gamma$ limits are translated into vertical speed $\dot{h}$ for presentation on the vertical speed tape on the right hand side of the PFD.

As described so far, this algorithm will work provided the $\left(V_{\text {crnt }}, \gamma_{\text {crnt }}\right)$-state falls well within the trim envelope. However, when this is not the case then the algorithm may fail to convey sensical information to the pilot. One such example, among many, occurs if the aircraft state is at location B, as shown in Fig. 66, In this case the trim envelope airspeed range does not exist because there are no trimmable airspeeds at the current flight path angle. To remedy situations such as this, our approach was to first establish an inside trim box, and then to always project the current aircraft state onto the box, as shown for point B in Fig. 6. before calculating the mapping to the PFD limits discussed in the previous paragraph.

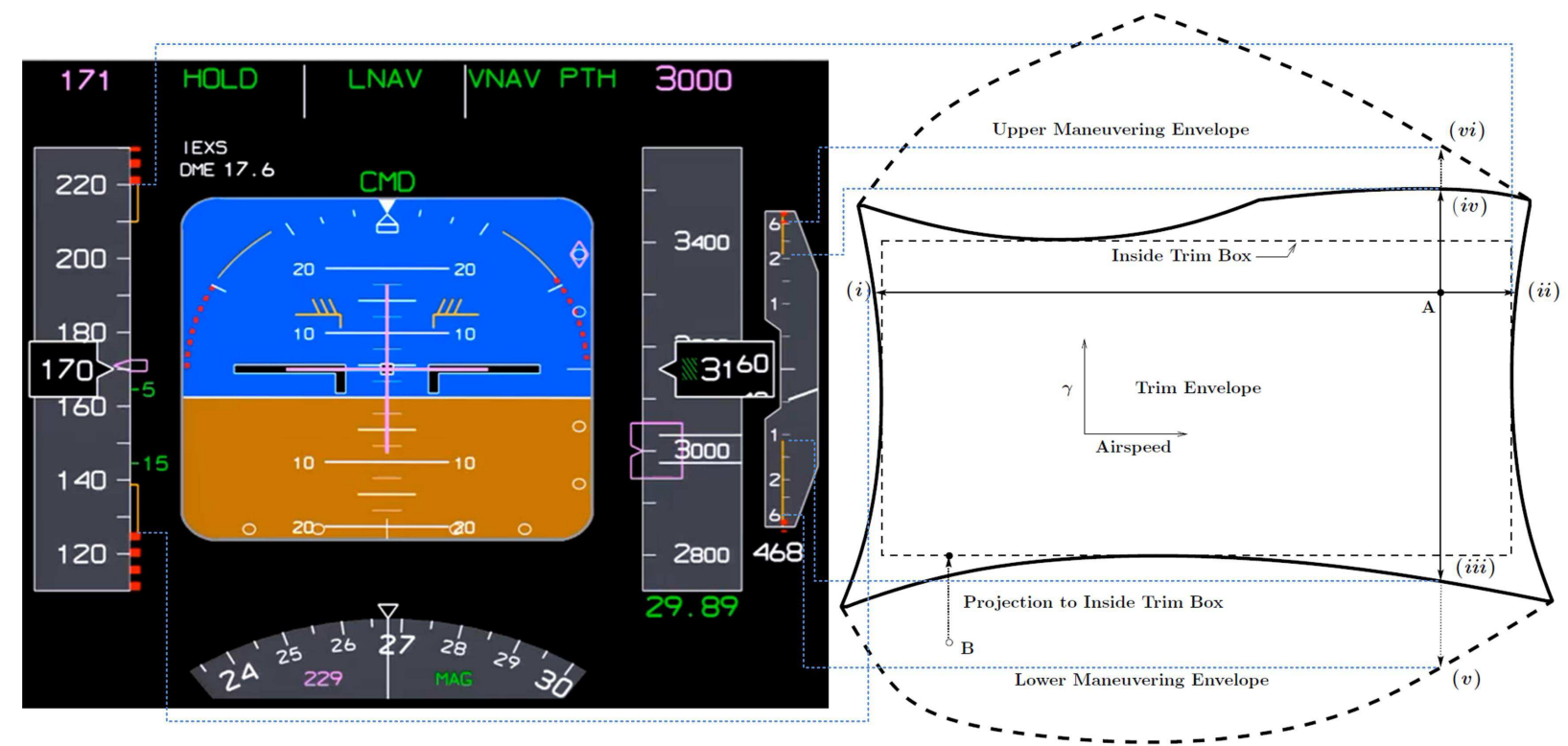

Figure 6. With the current aircraft state inside the trim envelope on the right side at A, the edges of the trim and maneuvering envelope are found and mapped to the PFD as follows: $(i)$ stall speed limit, (ii) structural overspeed limit, (iii) minimum steady-state vertical speed, (iv) maximum steady-state vertical speed, ( $v$ ) minimum maneuvering vertical speed, and $(v i)$ maximum maneuvering vertical speed. If instead, the current aircraft state is outside the trim envelope box at $B$, then the state is first projected onto the inside trim box prior to determining the PFD envelope limits, as described in the previous sentence.

When displayed in the manner shown in Fig. 6, the airspeed information is consistent with operational practice. Stall speed and overspeed are indicated by the red barber poles. The amber brackets have another specific meaning. The top of the lower amber bracket indicates the minimum speed at which a $35^{\circ}$ bank angle can be established without stalling the aircraft. When the airspeed falls within the lower amber bracket, then only bank angles less than $35^{\circ}$ are sustainable; and near the stall speed no bank angle is safe. On the other hand, the bottom of the upper amber bracket marks the maximum permissible airspeed at the next flap setting. Therefore, if the airspeed is in this upper amber region, then flap deployment to the next setting is unsafe. Current PFD technology, relies on a pre-calculated database, stored on-board, for this information. Table 2 shows the pre-computed maximum flap speeds (structural speed limit, at which the flaps are retracted automatically, the overspeed marker takes into account a safety buffer) used for the ACT model as an example. The technology developed in this paper, however, enables the online estimation of the limit information from the trim and maneuvering envelope calculations for the current aircraft configuration. This online information can be of critical importance in hazardous conditions such as icing.

The safe vertical speed limits displayed on the vertical speed tape provides new information, not available in current PFD technology. Like the airspeed tape limits discussed in the previous paragraph, the vertical speed limits are also conveyed using amber and red bands, indicating caution and danger, respectively. However, the reason that a particular aircraft state may be unsafe is of course different than it would be for 


\begin{tabular}{ccc}
\hline Flaps & overspeed marker IAS [kts] & automatic flap retraction IAS [kts] \\
\hline 1 & 240 & 278 \\
5 & 220 & 232 \\
15 & 210 & 220 \\
20 & 195 & 215 \\
25 & 190 & 210 \\
30 & 162 & 198 \\
\hline
\end{tabular}

Table 2. Typical pre-calculated IAS values for the flap dependent overspeed limits of the ACT model

the airspeed tape. On the vertical speed indicator, the amber regions mark the vertical speed ranges where no equilibrium can be established. In practice, this means that airspeed speed will increase in the lower amber region, even for idle thrust, and that airspeed will decrease in the upper amber region, even with full thrust. The red barber poles mark the regions outside of the maneuverability envelope. In practice, this means that it is impossible to return from this region to a trimmable aircraft state within $5 \mathrm{~s}$.

As with current technology, the operational use of the new limit information requires a safety buffer so that pilots have a chance to respond, before potentially loosing control of the aircraft. For the technology discussed in this paper, the appropriate margins are achieved by including margins in the limits of the range of possible values for the virtual inputs, which ultimately drive the envelope limits provided to the PFD. For the angle of attack $\alpha$, a safety margin of $2^{\circ}$ was provided, reducing the standard range $\alpha \in\left[0 ; \alpha_{\text {stall }}\right]$ to the restricted safe range $\alpha \in\left[0 ; \alpha_{\text {stall }}-2^{\circ}\right]$. For the elevator deflection $\delta_{e}$ an analogous safety margin of $1^{\circ}$ was provided, resulting in $\delta_{e} \in\left[\delta_{e_{\min }}+1^{\circ} ; \delta_{e_{\max }}-1^{\circ}\right]$.

\section{Improving the envelope limit resolution}

If the resolution used to compute trim envelope point grid is coarser than desired for the limit displays, then this can be improved for the specific trim envelope points used to drive the PFD indicators without significantly increasing the computation time through the use of a simple bisection method. For example, given a coarse calculation for $V_{\text {stall }}$, and trim envelope calculation resolution $\Delta V$, one can iterate

$$
V_{\text {stall }}=V_{\text {stall }}-\mathcal{I}\left(V_{\text {stall }}-\frac{\Delta V}{2^{k}}, \gamma_{\text {crnt }}\right) \frac{\Delta V}{2^{k}},
$$

for $k=1,2, \ldots, r$, and after $r$ iterations, $V_{\text {stall }}$ is inside the trim envelope and $V_{\text {stall }}-\Delta V / 2^{r}$ is not. Recall, $\mathcal{I}$ is the indicator function defined by Eq. (5). The appropriate version of this example can then be used to compute any trim envelope related limit to the improved resolution $\left(\Delta V / 2^{r}, \Delta \gamma / 2^{r}\right)$. This approach is significantly more efficient than computing all the points in the trim envelope at the higher resolution.

\section{B. Indication of the maximum pitch attitude angle}

The conventional maximum pitch attitude angle $\theta_{\max }$ maker position is yet another output provided by the envelope estimation algorithm. The marker corresponds to the pitch attitude angle $\theta$ where stall will occur. Since pitch attitude is $\theta=\gamma+\alpha$, its maximum value is given by

$$
\theta_{\max }=\gamma_{\text {current }}+\alpha_{\text {stall }} .
$$

This indicator tells the crew the pitch attitude angle that stalls the aircraft. Its expected use is primarily for manual flight operations.

\section{Indication of the maximum bank angle}

Maximum bank angle limits are provided along side the regular bank angle indicator on the PFD, as seen in Fig. 7 which is similar to the Airbus style PFD. The red bank markers show where stall will occur. 
The yellow bank markers are fixed at $\phi=35^{\circ}$, since nominal bank maneuvers for commercial aircraft stay within these bounds. However, as the aircraft slows, $\phi_{\max }$ eventually becomes less than $35^{\circ}$, and in this case the yellow markers collapse together with the red markers, as shown in Fig. 7(c), until no bank authority remains when the aircraft reaches the stall air speed.

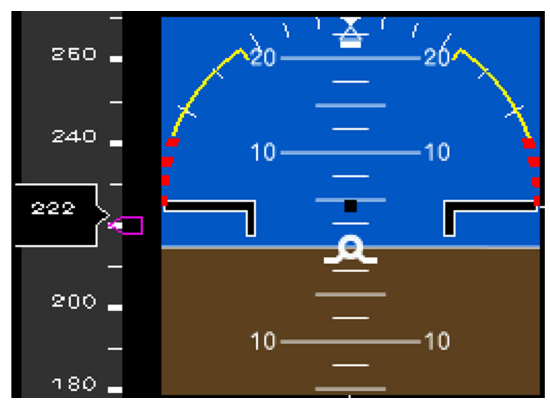

(a) indicated airspeed $222 \mathrm{kts}$

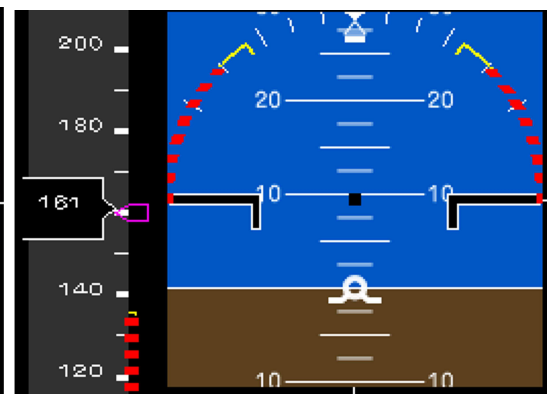

(b) indicated airspeed $161 \mathrm{kts}$

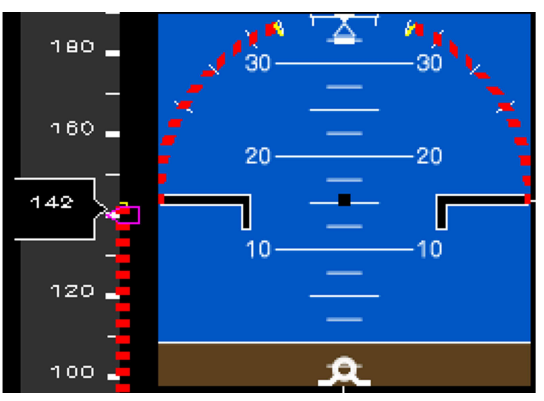

(c) indicated airspeed $142 \mathrm{kts}$

Figure 7. Maximum bank angle $\phi_{\max }$ of FLTz ACT model for different indicated airspeed values. No bank authority is left close to stall speed.

\section{Experiment method for the piloted simulation study}

The overall objective for the simulation study was to examine how crews managed their trajectory and energy state, both with and without new technologies, while interacting with the flight deck automation during relevant scenarios with air traffic control and other air traffic. Since the study involved several new technologies and a limited number of test subjects, its purpose was exploratory and its outcome was intended to guide future research and development efforts to elevate technology readiness. This section presents elements of the experimental method specific to the safe flight envelope aspect of the study. The details of the overall experiment are discussed separately in Ref. 19.

\section{A. Technologies}

In all, three new technologies were evaluated in the study:

1. Trajectory prediction. The Navigation Display (ND) and Vertical Situation Display (VSD) included a trajectory prediction with active drag estimation that shows the future trajectory of the aircraft out to 5 minutes if no action is taken by the pilots. An example is shown in Fig. 8 . The magenta line shows the programmed FMS route through the waypoints. The green line in both displays is the trajectory prediction.

2. Envelope estimation. On-board characterization of the safe maneuvering envelope, as presented in $₫$ and $₫$ above.

3. Predictive alerting. Combines the previous two new technologies to generate predictive alerts from an analysis of the trajectory prediction with respect to the maneuvering envelope. The predictive alerts were then displayed on the Engine Indication and Crew Alerting System (EICAS) display, ND, and VSD. Three types of alerts were provided: advisories for expected conflicts in 45 s were shown in white text on EICAS and in blue on ND/VSD, yellow caution messages alerted for conflicts in $10 \mathrm{~s}$, and red warnings indicated imminent danger. Example EICAS and ND displays with predictive alerts are shown in Fig. 9

See Refs. 19 and 20 for more information about the new trajectory prediction and predictive alerting features. 
The baseline display configuration for the study was the conventional cockpit configuration without any of the above new technologies. The VSD, without the green trajectory prediction was also considered part of the conventional cockpit configuration, despite the fact that many test subject pilots were not familiar with this kind of display.
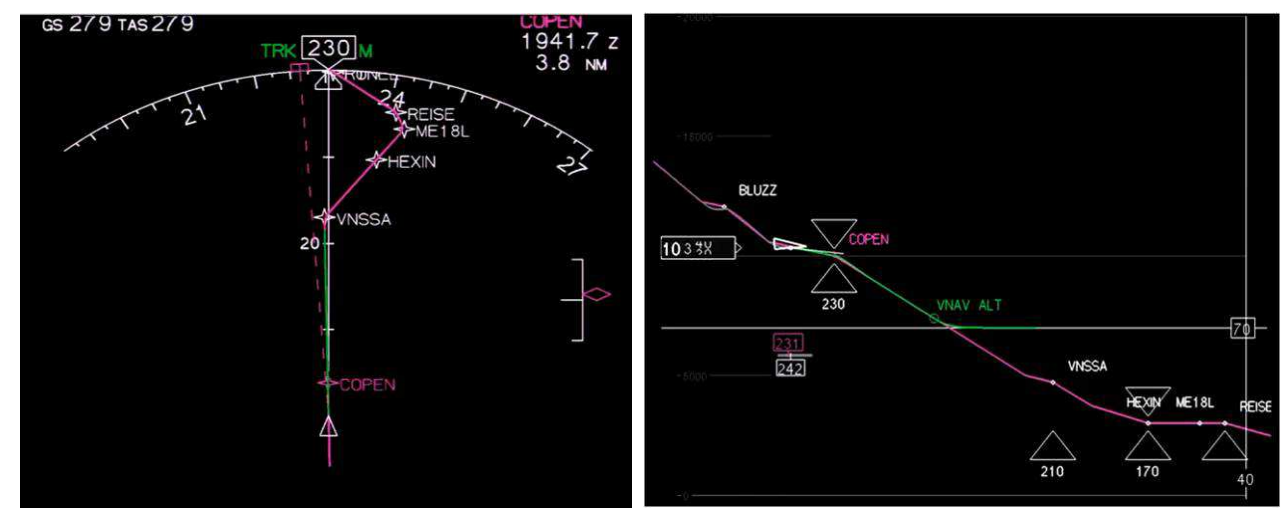

(a) Navigation Display with predicted trajec- (b) Vertical Situation Display with predicted tratory jectory

Figure 8. Predicted trajectory information on the flight deck
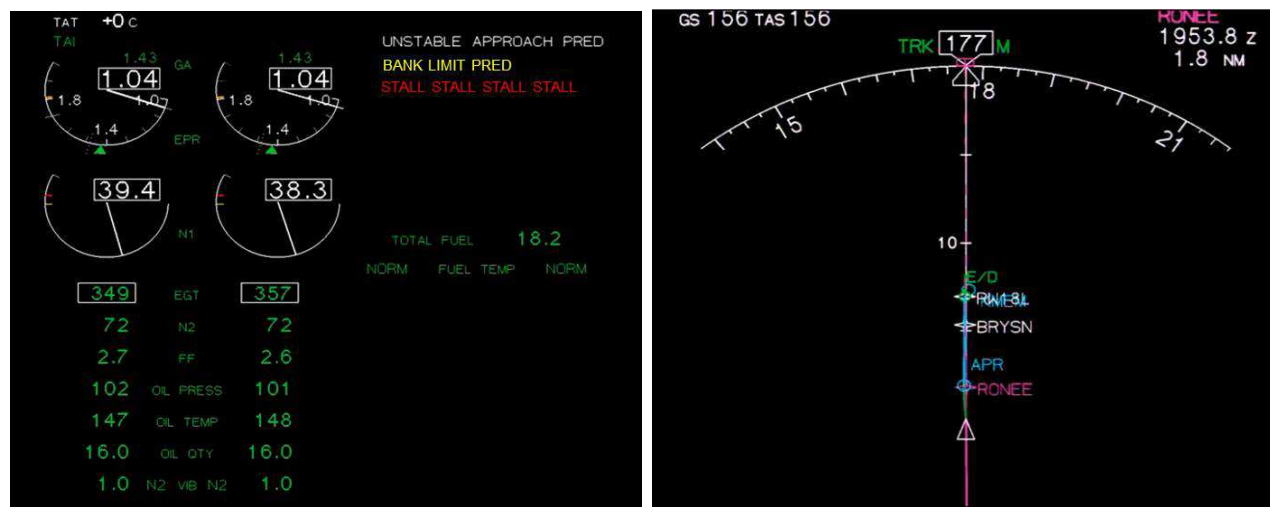

(a) Engine Indication and Crew Alerting System (b) Navigation Display with predictive alerting display with predictive alerting

Figure 9. Predictive alerting information on the flight deck

\section{B. Scenarios and procedures}

Four challenging descent scenarios were designed for the study, all of which were inspired by relevant air accidents and incidents from the aforementioned CAST study 2 The scenarios were flown in a randomized order designed so that half the pilots flew with the baseline technology first, and the other half flew with the new technology first. Two of the scenarios were relevant for the envelope research. These were the icing and the unscheduled stabilizer trim scenarios detailed below. In the the icing scenario, higher minimum airspeeds were required to prevent stall, while in the unscheduled stabilizer trim scenario, lower maximum airspeeds prevented elevator saturation. Information about the two other scenarios not discussed here is available in Ref. 19.

Prior to beginning the evaluation, the pilots were given a one hour briefing that covered an overview to the experiment, its objectives, as well as the baseline and new technologies. The crews were made aware 
they would be flying challenging descent and landing scenarios, but no specific details were provided. The pilots were also given an hour for two training runs in order to gain some familiarity with the simulator, aircraft model, and experiment procedure.

Each test scenario was initialized with the autopilot and flight management system (FMS) completely preprogrammed. The pilot flying had the choice to navigate by means of the FMS, the mode control panel (MCP), or manually, as he deemed appropriate in the scenario at hand. Prior to the start of each simulation run, the crew was given an ATC clearance, and was subsequently vectored by air traffic control during their approach. The simulations included other air traffic as well.

\section{Icing scenario}

In specific atmospheric conditions, ice accretion is possible on the aircraft wings. In this scenario, it was assumed that the regular thermal anti-ice systems, which are conventional equipment on all civil airliners, were operating but were incapable of removing the ice build up on the wings. The goal of this scenario, then, was to see how wing icing impacts the maneuvering envelope, and to see if displaying the new envelope information to the pilots helped them to maintain safer flight operations.

In this scenario, the aircraft was initialized in an icing condition with modified flight dynamics. Ice accretion on the wings causes the wings to provide less lift, to experience more drag, and to stall for smaller angles of attack. To simulate these effects, the lift and drag curves for the aircraft were modified as shown in Figs. 10(a) and 10(b),

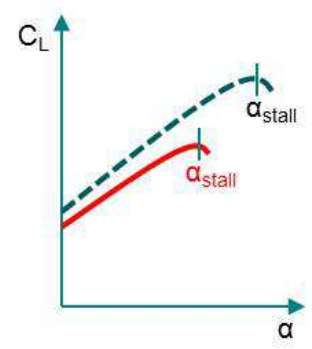

(a) Effect of icing on lift (b) Effect of icing on drag curve

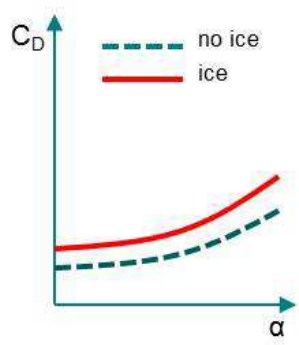

curve

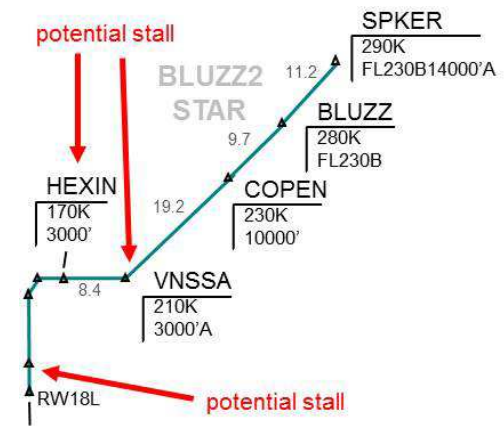

(c) Modified standard instrument arrival (STAR) over BLUZZ to RW18L

Figure 10.

At the start of the simulation run, the flight crew was informed about the icing condition by an 'icing wing' caution message shown on the EICAS display. The top left corner of this display also showed that Thermal Anti-Ice (TAI) was working. The aircraft was set up to fly the published profile along the BLUZZ2 STAR arrival to runway 18L, as shown in Fig. 10(c), Deceleration segments were included in the STAR ahead of waypoints COPEN, VNSSA and HEXIN. As a consequence of the icing, there were larger than expected speed overshoots during the deceleration segments. At waypoint VNSSA, there was a deceleration to $210 \mathrm{kts}$ in combination with a turn, followed by a further deceleration to $170 \mathrm{kts}$ at HEXIN. At both of these waypoints, there was the potential for stall to occur, unless the crew deployed flaps earlier than expected for nominal scenarios.

\section{Unscheduled stabilizer trim scenario}

Another scenario considered in this study, was an 8 degrees nose down unscheduled stabilizer trim position (the maximum value in flight), which generated a pitch up moment. The aircraft was initialized in this condition. The disturbing moment then had to be counteracted by the elevators, which came close to their 
saturation limits, especially during a go-around. This resulted in significantly stricter upper envelope limits on speed and climb rate.

At the start of the run, the flight crew was informed of the stabilizer issue, by an 'unsched stab trim' EICAS message. The lower EICAS display showed the nose up stab trim at $8^{\circ}$ and a compensating elevator deflection of more than $14^{\circ}$, which was close to the saturation limit of $15^{\circ}$. The BRBBQ2 STAR arrival to runway $18 \mathrm{R}$ was flown in this scenario, as shown in Fig. 11(a) At waypoint SAAMM, the flight crew received an ATC instruction to go-around due to traffic on runway, shown as phase (1) in Fig. 11(b). The pilot performed a go-around by increasing thrust and establishing a positive rate of climb with gear up in phase (2). There were three options available for the execution of the go-around: using a switch on the MCP, flying manually but using auto-throttle in takeoff/go-around (TOGA) mode, or full manual control for side-stick and throttle. This flight regime (high speed, high thrust, steep climb), combined with the nose down stabilizer trim position caused a large pitch-up moment that risked exceeding of the pitch-down elevator authority at (3). As a consequence, the maximum speed and climb rate should have been limited during go-around to avoid elevator control saturation.

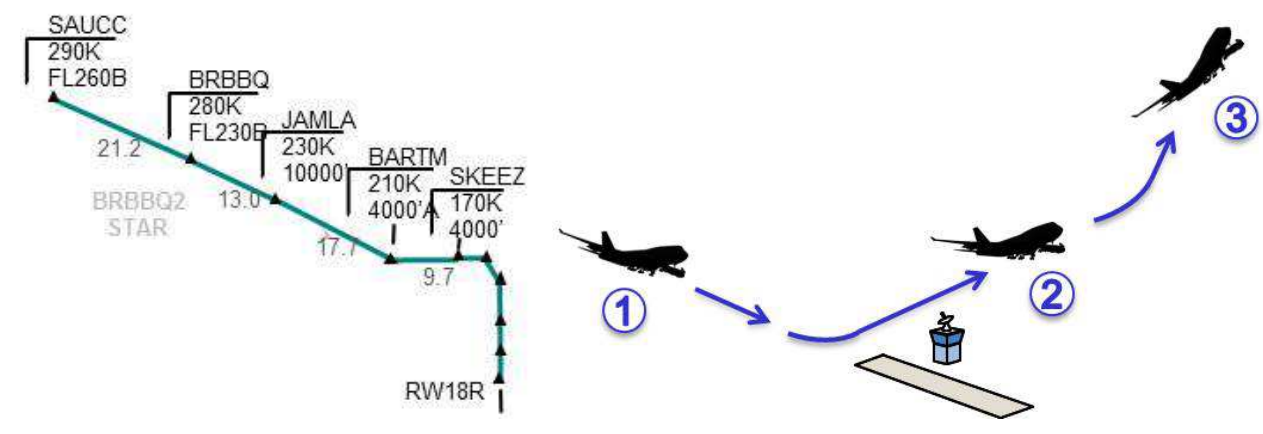

(a) Modified standard instrument arrival (STAR) over BRBBQ to RW18R

(b) Sequence of events during go-around

Figure 11. Setup of the unscheduled stabilizer scenario

\section{Apparatus}

The Advanced Concepts Flight Simulator (ACFS), shown in Fig. 12 is a six degree-of-freedom research flight simulator with configurable flight deck instrumentation systems, a $180^{\circ}$ field of view outside visual display system, a hydraulic motion system, as well as digital sound and aural cueing systems. ${ }^{21}$

\section{Flight Deck Instrumentation}

The flight deck was set up to resemble a generic two-person cockpit of a widebody airliner. The installed hardware consisted of two aircraft seats, hydraulically actuated sidesticks, rudder pedals, a control pedestal, six large and two small LCD screens to display the flight instruments, and a Boeing style mode control panel. The displays were based on the B767-300 electronic flight instrumentation system and were rendered using OpenGL running at a $60 \mathrm{~Hz}$ refresh rate.

\section{Outside Visual System}

The out-the-window visual scene was provided by a Flight Safety Vital X PC-IG system, configured with SkyLight 1500 raster/calligraphic projectors in three channels. The resulting system produced a seamless, edge-blended, cross-cockpit image with a 180 degree horizontal and 40 degree vertical field-of-view. Day, dusk, and night scenes were available with databases and weather simulation capabilities as required. The projector refresh rate was $60 \mathrm{~Hz}$. For this evaluation, a visual representation of Memphis International Airport was used. All runways and major taxiways were in their correct location, along with the most 


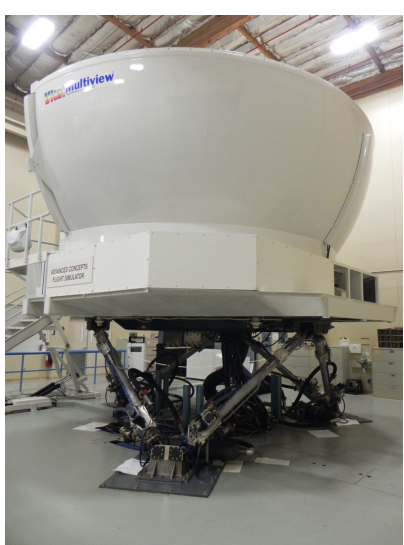

(a) Outside view

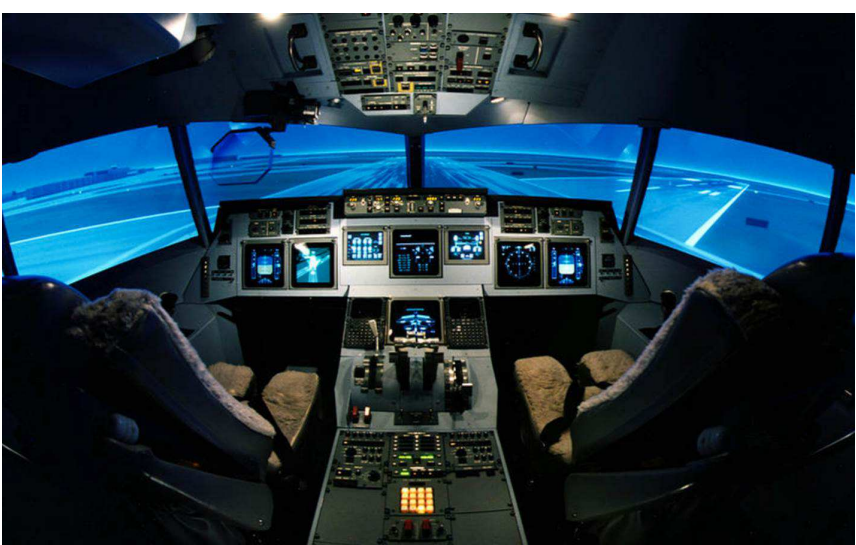

(b) Cockpit view

Figure 12. Advanced Concepts Flight Simulator at NASA Ames Research Center

important buildings on the airfield. The surrounding area was kept simpler, with a textured ground plane showing a rough outline of Tennessee.

\section{Motion System}

The motion cues were provided by a six degree-of-freedom synergistic system. The hydraulic servo-actuated system was originally designed to meet or exceed the performance specified by MIL-STD-1558 for the rated payload.

\section{Participants}

Twenty commercial airline pilots (from three different carriers) participated as ten two-person crews, with each crew consisting of pilots from the same carrier. The participating commercial flight crew members were between 43 and 61 years old, with an average age of 56 years. Their flight hours were between 6,000 and 23,000 , with an average of $14,700 \mathrm{hrs}$. All of the crew members were either current on the Boeing $757 / 767$, or had flown this type of aircraft earlier in their career.

\section{Results}

Given the exploratory nature of the experiment and that several technologies were evaluated it is not possible to provide strong statistical conclusions on any particular aspect of the experiment, but the data was sufficient to observe certain trends. The evaluation results for the icing scenario are discussed first in subsection $\mathrm{A}$ and the unscheduled stabilizer scenario is analyzed in subsection B In each subsection, the results from one particular crew are discussed first, and are then followed by a performance analysis that covers all of the crews together.

The envelope limits for both current technology and new technology displays are discussed in this section. Current technology display limits are a function of flap setting only and are read from precomputed data tables stored onboard the aircraft. The new technology limits are updated online based on sensor and system identification information in addition to aircraft configuration changes, which include flap setting, bank angle, gear position, speed brake extension, and stabilizer position. The new technology limits therefore represent actual limits for the flight condition of the aircraft, and are referred to as actual limits in the discussion below. It is understood that the actual limits are still estimates in this context. 


\section{A. Icing scenario}

The envelope bounds for IAS and bank angle are shown in Fig. 13 for a particular approach trajectory under icing conditions. The x-axis is plotted with respect to along track distance (ATD), so that one can observe the impact of the deceleration segments, discussed in $\{[\mathrm{IV} \mid \mathrm{B}] 1]$ on the actual vertical path. The two graphs in the left column show the path flown with current technology. For current technology scenarios, the magenta lines indicate the airspeed limits presented to the crew, which are based on precomputed data tables that do not account for the the influence of icing. The red airspeed and bank limits are the actual limits computed by the envelope estimation method discussed in this paper. These actual limits are not displayed on the PFD for the current technology scenarios. Conversely, the graphs in the right column represent new technology scenarios, where the actual airspeed and bank angle limits, accounting for the influence of icing, are displayed on the PFD for the pilots. The solid red lines on the IAS graphs correspond to the stall airspeed limit, taking into account a safety buffer of $2^{\circ}$ for the stall angle of attack $\alpha_{\text {stall }}$. The dashed lines on the IAS graphs show the maneuvering limits, defined as the speed where stall will occur in conjunction with a $35^{\circ}$ bank. The dashed lines on the bank angle plots are fixed at $35^{\circ}$, while the solid red lines represent the bank angle where stall will occur for the current airspeed. One may further observe in Fig. 13(c) that the solid bank angle limit lines overtake the dashed limit lines when the indicated airspeed exceeds the maneuverability speed limit shown in Fig. 13(a)

As one should expect, the maneuvering and stall speed limits for the new technology scenarios are significantly higher due to the influence of icing. Higher speeds are required in turns to compensate for the reduced vertical component of the tilted lift vector. It can be seen in the current technology scenario that the crew approached the actual stall speed much closer without knowing it. The second deceleration segment and simultaneous banking brought them to the verge of stalling (taking into account the safety buffer) without being aware of it. The third deceleration segment brought them very close to stall again, but flaps were deployed just in time to avoid it. In the bank angle graphs, one further observes that speed variation and flap deployment, affecting $C_{L_{\max }}$ directly, have a direct influence on the maximum bank angle. In the new technology graphs, the crew clearly relied on the more accurate maneuvering and stall speed limits to stay further from stall limit, and to even secure the full $35^{\circ}$ bank authority for nearly the entire duration of the flight.

Figure $14(\mathrm{a})$ shows the margin between the current IAS and the stall limit $V_{\min }$, in addition to the margin between the current angle of attack $\alpha$ and the safe limit angle of attack $\alpha_{\text {safe }}$, which is $2^{\circ}$ smaller than $\alpha_{\text {stall }}$. The $\alpha$-margin includes the influence of the flap setting on the stall limit, shown in Fig. 14(b)] This figure also shows that, as a general rule, $\alpha$ increases during deceleration segments and decreases with flap deployment.

The bar plot appearing in Fig. 15 shows the minimum airspeed and angle of attack margin that occurred across the full simulation run for each individual crew. Within each plot, the set of bars on the left cover the runs with current technology, and the set of bars on the right cover the runs with new technology. For example, Fig. 14(a) shows that crew A violates the limits briefly with current technology, but maintains a significant margin with new technology. Analyzing the data for all of the crews shows that six out of ten crews exceeded the limits with current technology, without being aware of it. On top of each set of bars, the average and standard deviation across the bars are indicated. The standard deviation values were further corrected for variability between subjects. Figure [15] confirms that, looking at the whole test group, more safety margin is maintained with respect to the envelope boundaries when the new technologies were available. These results are statistically significant, but one has to take into account that the test group consists of only 10 crews.

The flap deployment strategy in the icing scenario is shown in Fig. 16. This figure shows the average indicated airspeed for each flap setting over the entire simulation run, for all crews. The standard deviations were corrected for variability between the individual subjects. Comparing strategies with and without new technology, shows that the crews deployed flaps at higher speeds with technology, since they were aware of the actual stall limits accounting for the influence of icing.

Summarizing for the icing scenario, the safe envelope boundaries presented to the crews, were consistent with maneuvers and airplane configuration changes (intentional as well as unintentional). When the safe envelope boundaries were presented to the crews, they adapted their flap deployment strategy. By deploying 


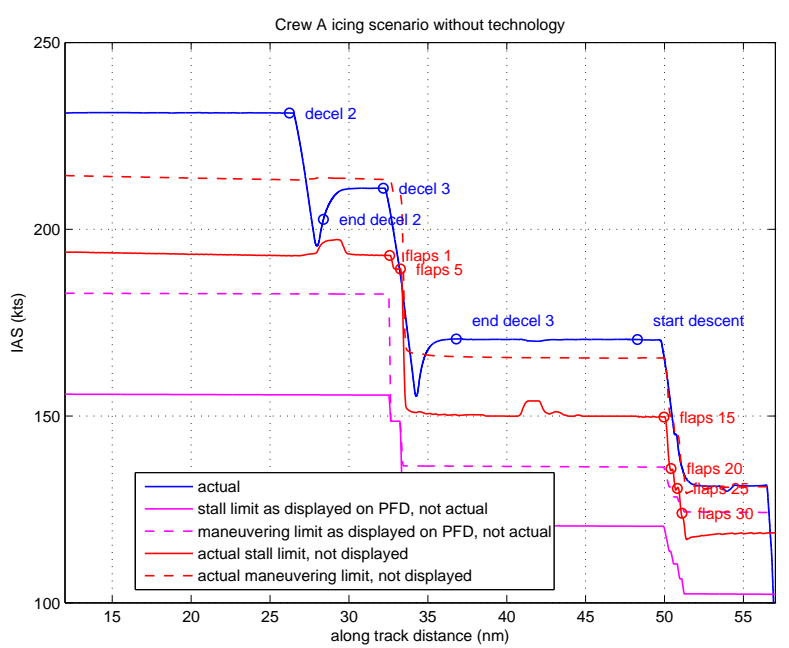

(a) IAS current technology

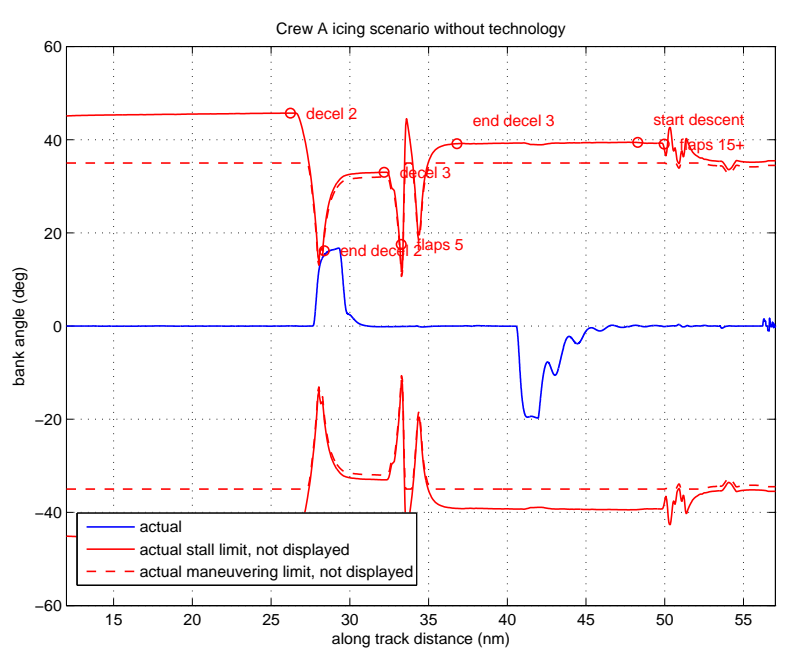

(c) bank angle current technology

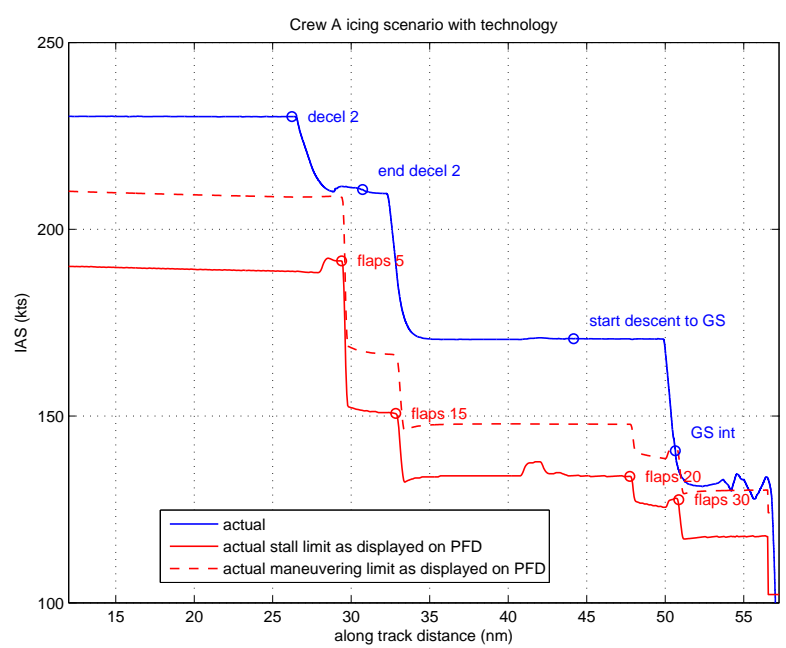

(b) IAS new technology

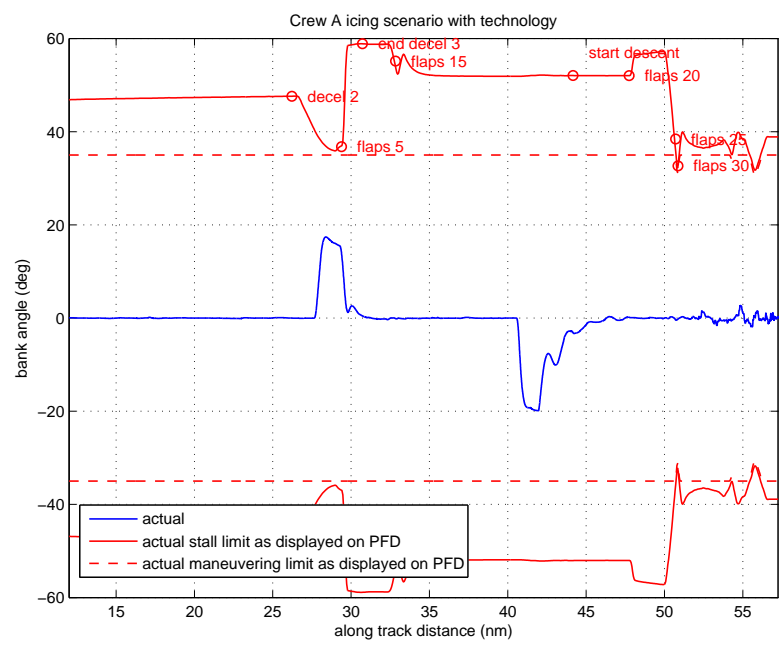

(d) bank angle new technology

Figure 13. Along track distance evolution of envelope lower bounds of indicated airspeed $V_{I A S}$ and bank angle $\phi$ for icing scenario, comparison of current technology and new technology for crew A. 

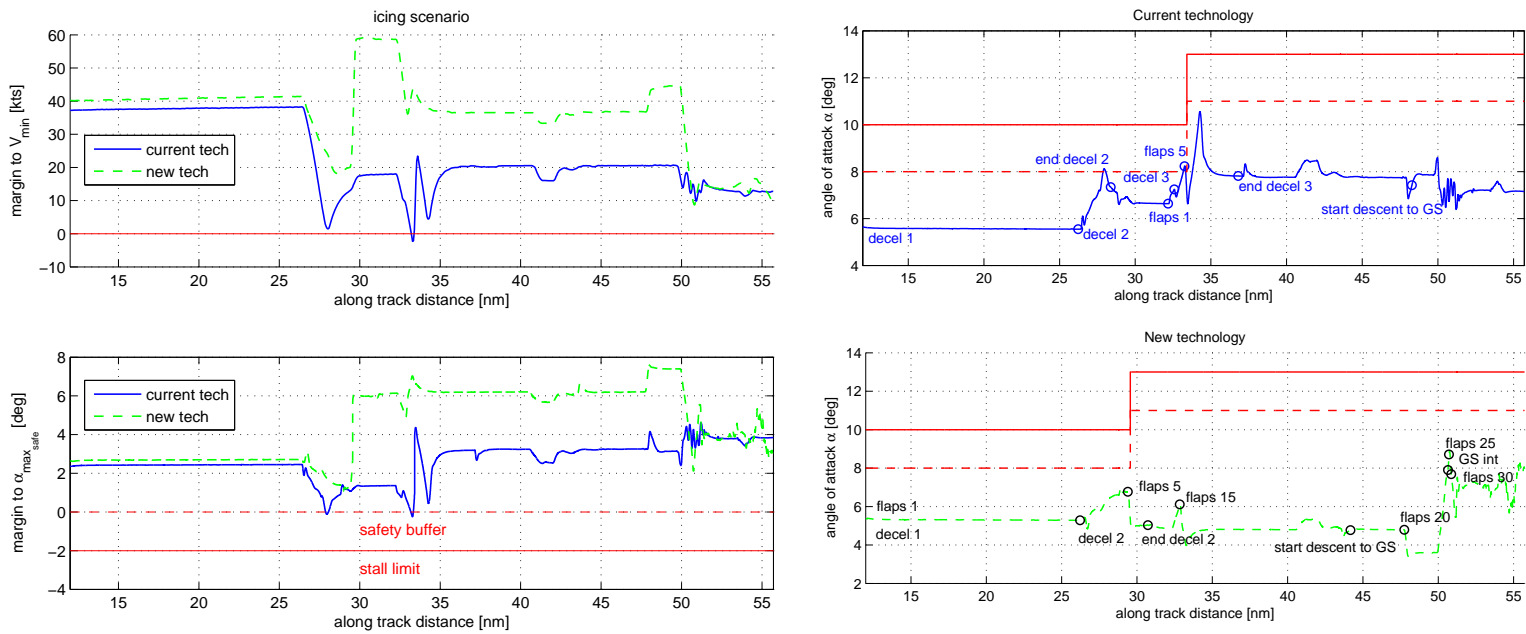

(a) IAS and $\alpha$ margins

(b) $\alpha$ and its upper bound

Figure 14. Along track distance evolution of indicated airspeed and angle of attack margins for the icing scenario with current and new technology, crew A.
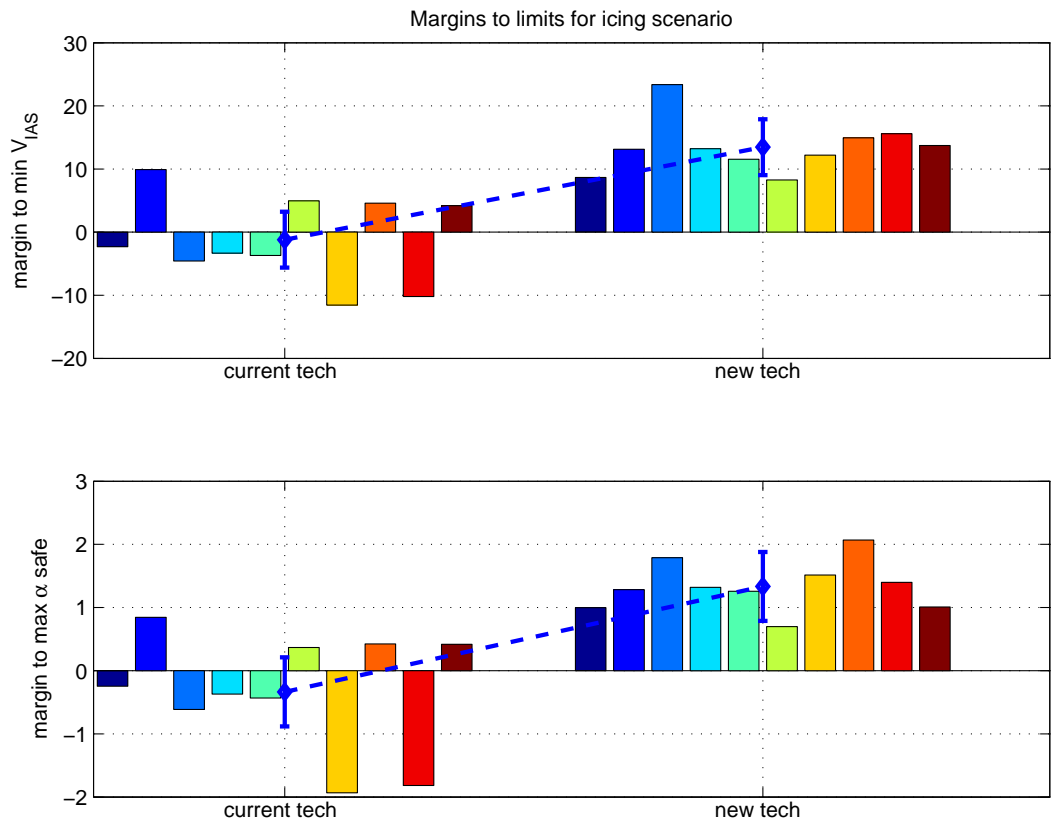

Figure 15. Statistical analysis of minimum margins to envelope boundaries for icing scenario, all crews 


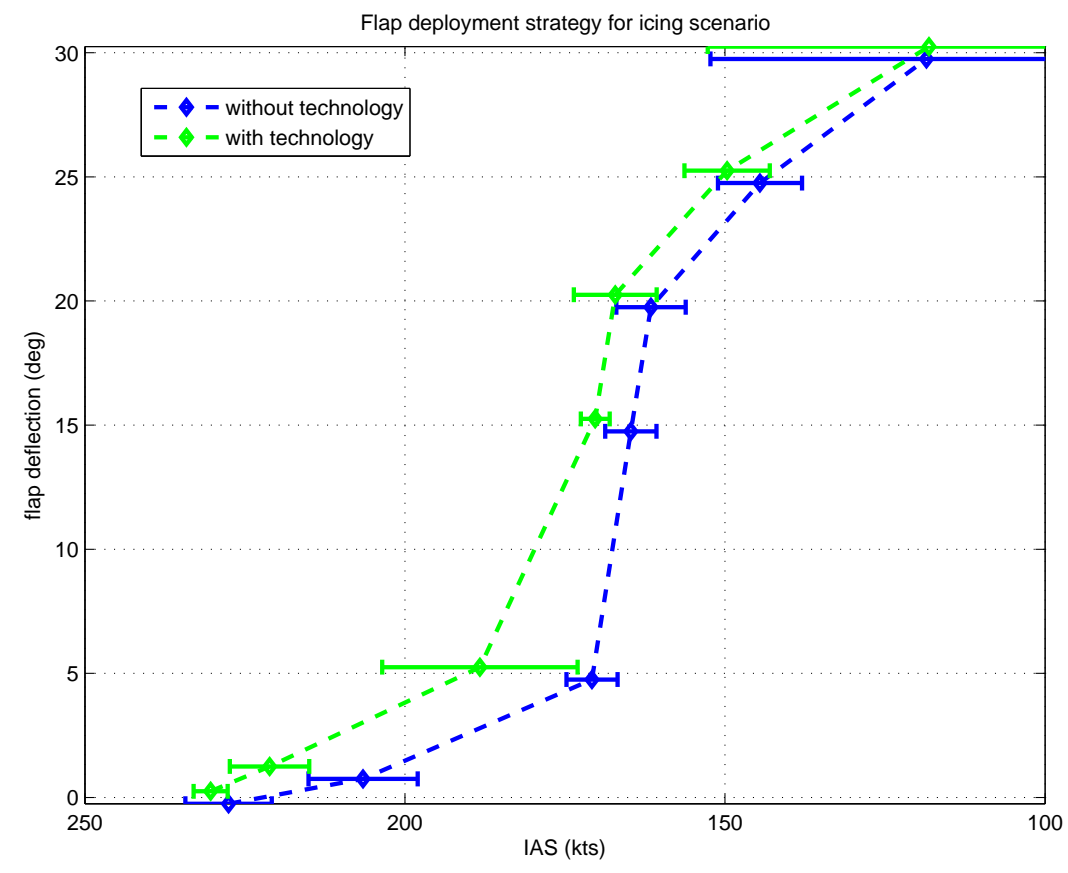

Figure 16. Flap deployment strategy in icing scenario, all crews

flaps earlier in the descent for higher airspeeds, they maintain significantly larger safety margins to the actual flight envelope boundaries for the aircraft with wing icing.

\section{B. Unscheduled stabilizer trim scenario}

The results for the unscheduled stabilizer scenario provided a number of insights into the relevant dynamics and effect of the envelop limit indicators during a go-around maneuver.

Figure 17 shows the time histories of the envelope upper bounds for IAS and climb rate in the go-around part of the unscheduled stabilizer scenario. As with the icing scenario, the two graphs in the left column show the path flown with current technology. For current technology scenarios, the magenta lines indicate the airspeed limits presented to the crew on the PFD. These are based on precomputed data tables that do not account for the influence of the unscheduled stabilizer. The red speed and climb rate limits are the actual limits, which are not displayed on the PFD for the current technology. The graphs on the right hand side represent the new technology scenario, where the actual limits for speed and climb rate are displayed on the PFD, taking into account the influence of the stabilizer position. The solid limit line in the speed graph corresponds to the overspeed limit, taking into account a safety buffer of $1^{\circ}$ for the elevator deflection $\delta_{e}$. The dashed limit line in the speed graph is the overspeed for the next (higher) flap setting. The dashed limit in the climb rate graph represents the flight condition where the throttle saturates providing maximum thrust $T_{\max }$. Higher climb rates $\dot{h}$ will lead to a loss in indicated airspeed IAS. The solid line represents the flight condition from where it will take at least $5 \mathrm{~s}$ to reach a steady state flight condition, or which will take at least $5 \mathrm{~s}$ to be reached starting from any steady state flight condition, or where control authority is lost. For example, in the unscheduled stabilizer scenario, the red line marks where the elevator will saturate, and thus cannot compensate any longer the moment induced by the misaligned stabilizer, which then results in an uncontrollable pitch up moment.

Referring back to Fig. 4 , one expects significantly lower overspeed limits due to the influence of the stabilizer. For current technology, the displayed speed limits vary only with flap setting, while the new technology limits are more accurate because they account for things like variation in speed and climb rate, 


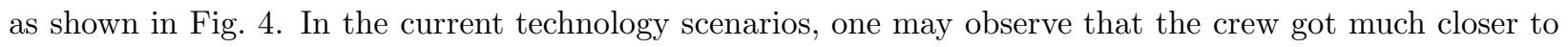
the actual overspeed limit without knowing it. Just before selecting flaps 15, the crew came to the verge of overspeeding at the flaps 25 setting (taking into account the computational safety buffer and the structural buffer) without awareness of the additional aircraft limitation at that setting. Selecting flaps 15 helped them to increase their speed and climb margin, however it is not clear if this action was intentionally taken with this purpose in mind.

In the vertical speed graphs, the maximum climb rate is directly influenced by speed variation (higher maximum vertical speed for higher speeds) and flap retractions (significant reduction in maximum climb rate for flap setting 5 and less). The huge dip in the climb limit in Fig. 17(c) is the consequence of exceeding the overspeed limit in Fig. 17(a), since this is a two dimensional upper limit for speed and climb rate, as shown in Fig. 4. Especially for the new technology, it can be seen that there is no significant speed increase in Fig. 17(b) when the vertical speed exceeds the steady climb limit in Fig. 17(d) However, this trend is less apparent when one compares Fig. 17(a) and 17(c), The new technology graphs show that the crew relied on the more accurate overspeed limits. They exceeded the maximum steady climb limits only marginally, and this did not seem to lead to any unsafe situations during the go-around maneuver.

Figure 18 focuses on the margins of the indicated airspeed to its overspeed limit $V_{\max }$, the vertical speed $\dot{h}$ to its maximum value $\dot{h}_{\max }$, and the input elevator deflection $\delta_{e}$ to $\delta_{e_{\max }}$, which is considered $1^{\circ}$ smaller than its real value as a safety buffer. The elevator deflection $\delta_{e}$ signal contains high frequency components, and is directly steered by the pilot when in 'command' (CMD) mode, or otherwise in autopilot (AP) mode. In order to compare this signal meaningfully with indicated airspeed and vertical speed (which depends on flight path angle $\gamma$ and speed) margins in similar bandwidth ranges, the elevator margin needs to be low-pass filtered with the appropriate time constants of a.o. $\gamma$. The figure shows that in the current technology scenario, when indicated airspeed and vertical speed boundaries are violated, then the elevator safety limit is exceeded as well. However, elevator saturation can also occur without any violations of IAS and $\dot{h}$ limits, as one can observe around $t=1020 \mathrm{~s}$ and $t=1060 \mathrm{~s}$. Data analyses of other participants lead to similar observations. Exceeding the IAS and $\dot{h}$ bounds also does not necessarily imply that the elevator will saturate. There are a few possible explanations for this observation. The additional trim criterion, in this specific context, uses the higher bandwidth pitching moment $M$ from time scale separation diagram in Fig. 1(b), Moreover, the envelope boundaries for this trim criterion are calculated according to the discussion in $₫ \mathrm{IIE}$, which relies on the corresponding trim values of the virtual inputs $\alpha$ and $T$ for every steady state flight condition $V, \gamma$. However, the go-around maneuver is very dynamic an as such requires actual inputs for $\alpha$ and $T$ that may differ significantly from the values required to hold a trim condition. Also the accuracy of the identified values for the aerodynamic coefficients related to the dimensionless pitching moment coefficient $C_{m}$ during the dynamic go-around maneuver may play a role in this context. This is a topic for further research.

The bar plot in Fig. 19] shows the minimum margins to the saturation limit of the elevator deflection $\delta_{e}$ for the go-around portion of the simulation run for each individual crew. This was done for current technology on the left, and for new technology on the right. Due to the complexity of options crews made a variety of different decisions that would have affected the results. For example, crew B violates the limits with current technology, but maintains a small margin with new technology, see Fig. 18. The statistical analysis of the margins for IAS and $\dot{h}$ was not included since there was no accurate and consistent link with the elevator, which is in contrast to the margins for the icing scenario. With respect to the margins maintained for IAS and $\dot{h}$, the data also show a significant learning effect between first and second run, irrespective of which technology is considered first. Analyzing data for all crews and comparing current technology versus new technology shows that six out of ten crews improved their minimum margins to elevator saturation, while three crews decreased their margins. For one crew there was barely a difference. On top of the bar plots, the average values are indicated as well as their standard deviations, which were corrected for variability between subjects. Figure 19 confirms that, looking at the whole test group and despite the earlier side-notes, more safety margin is maintained with respect to the saturation limit with new technologies.

The climb out profile in the go-around phase of this scenario is shown in Fig. 20. This figure shows the average along track distance for each $500 \mathrm{ft}$ of altitude change during the go-around, for all crews. The standard deviations were corrected for variability between the individual subjects. Comparing the average 


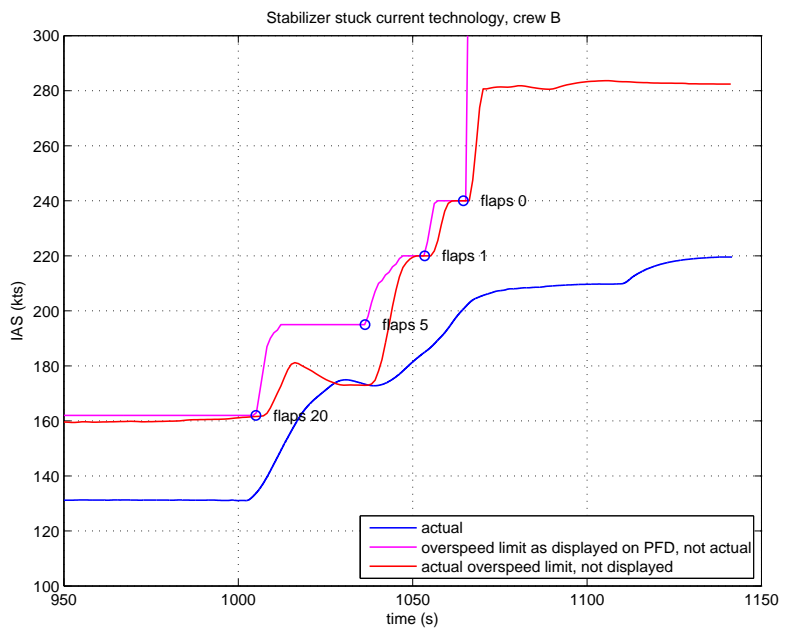

(a) IAS current technology

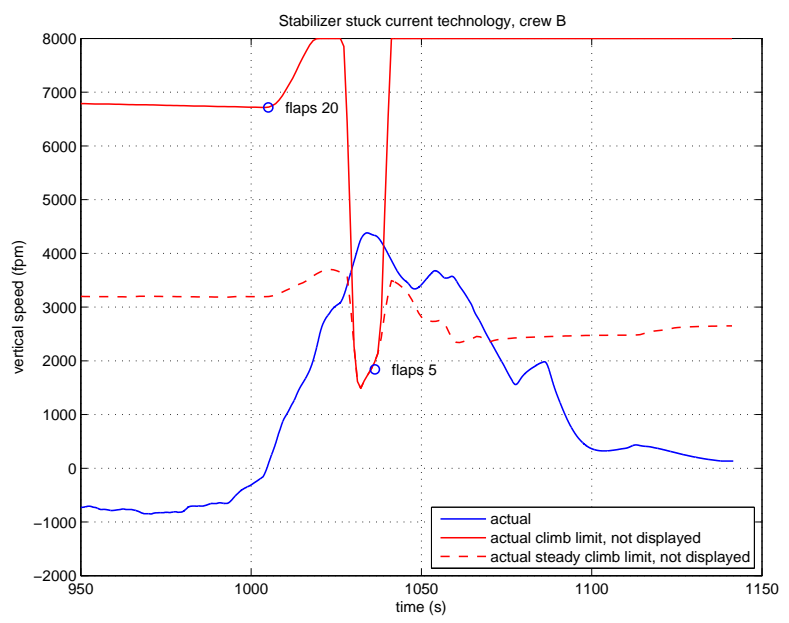

(c) $\dot{h}$ current technology

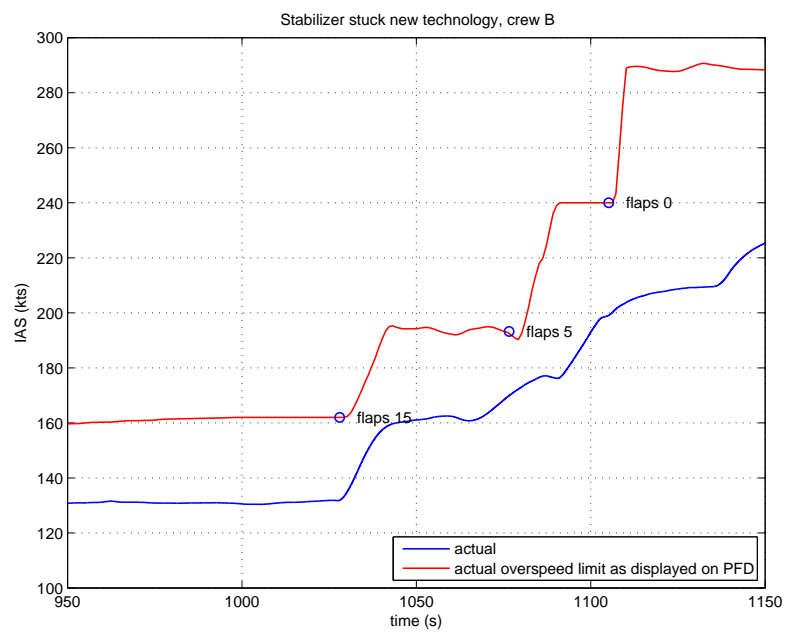

(b) IAS new technology

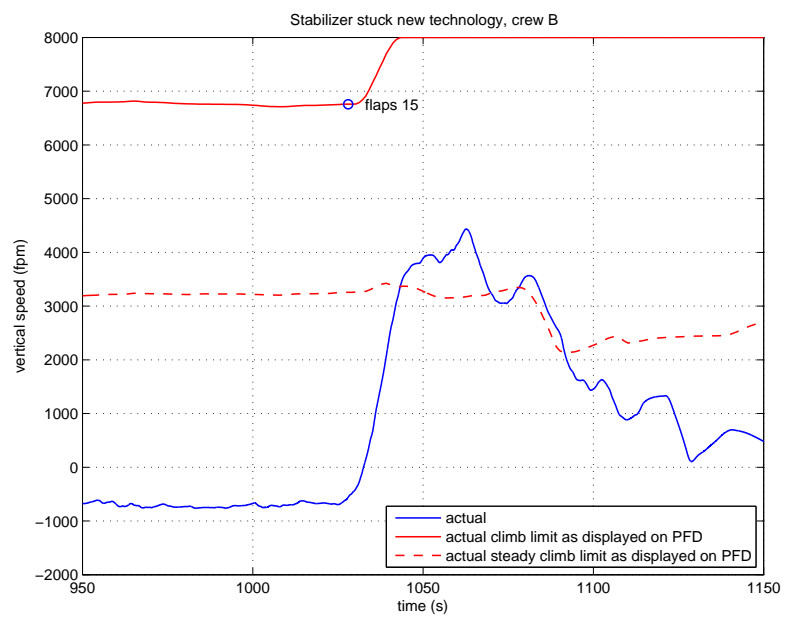

(d) $\dot{h}$ new technology

Figure 17. Time histories of envelope bounds of indicated airspeed $V_{I A S}$ and vertical speed $\dot{h}$ for unscheduled stabilizer scenario during go-around, comparison of current technology and new technology for crew B. 

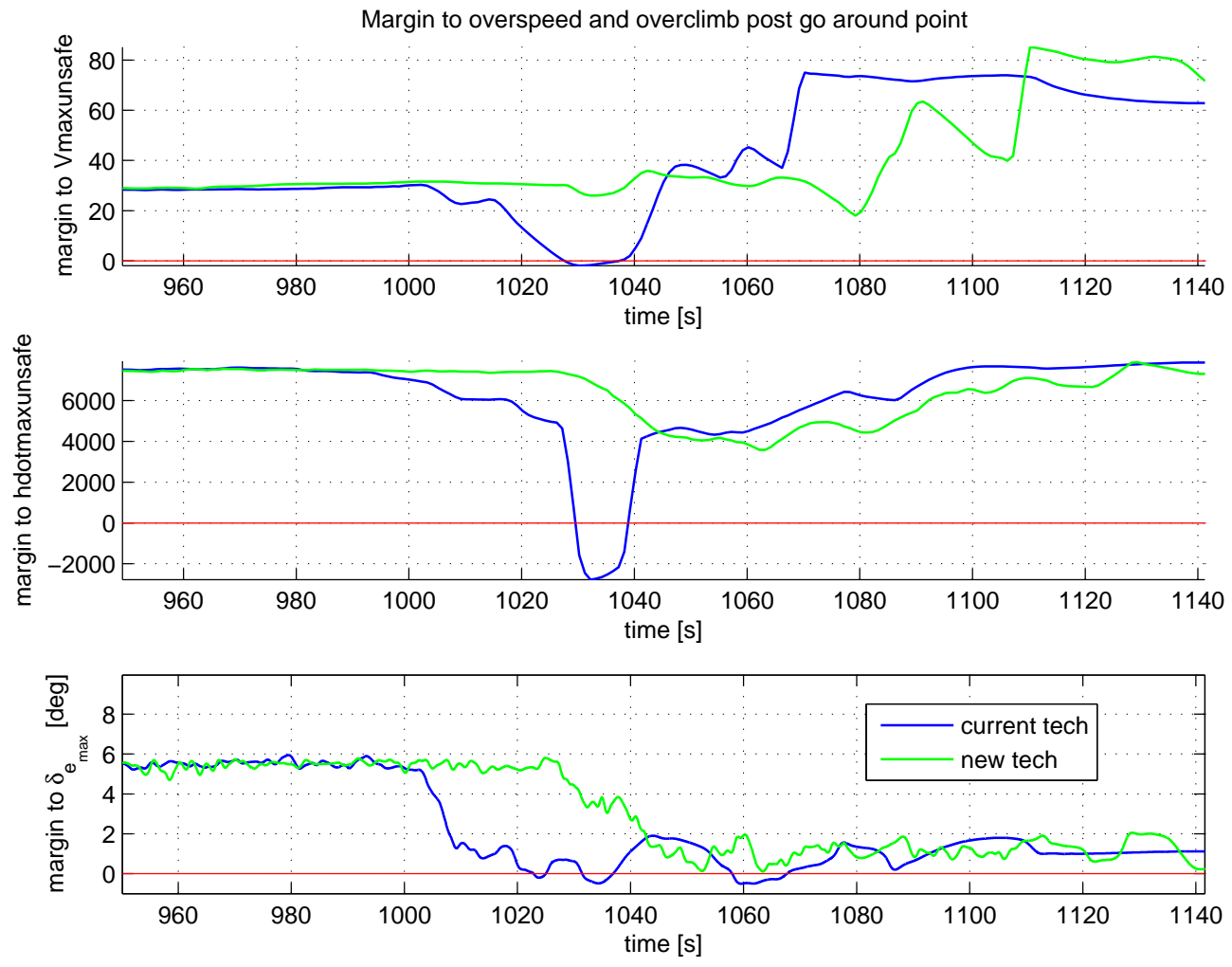

Figure 18. Time histories of indicated airspeed, vertical speed and elevator deflection margins during go-around for the unscheduled stabilizer scenario, crew $B$

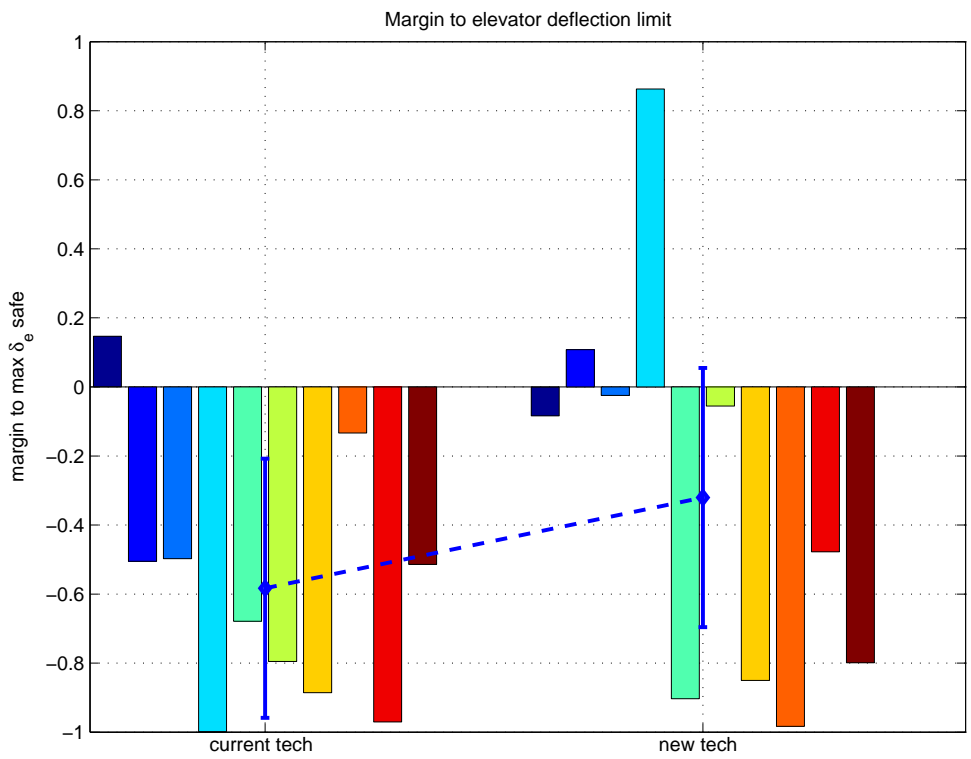

Figure 19. Statistical analysis of elevator margin to deflection limit during go-around for unscheduled stabilizer scenario, all crews 


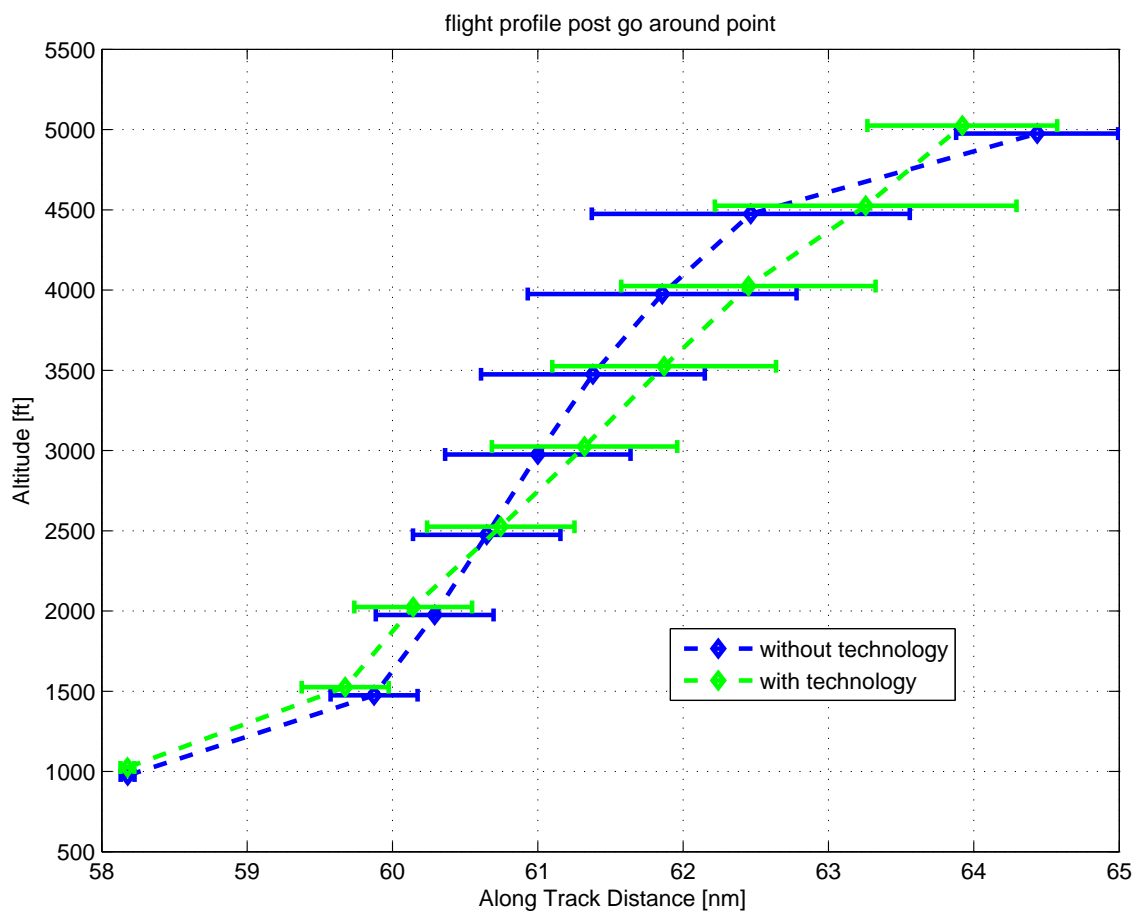

Figure 20. Climb out profile during go-around maneuver, all crews

ascent trajectories in Fig. 20, with and without technology, shows that the crews had a reduced (safer) average climb rate with technology, since they were aware of the actual overspeed and steady climb rate limits that reflected the influence of the stabilizer.

The variety of outcomes evident in Fig. 19 along with the variability that appears in Fig. 20 can be attributed to the following factors. First, as mentioned in $\$ \mathrm{IV}|\mathrm{B}| 2$, pilots were able to choose between three methods for controlling the go-around: manual steering, full autopilot control with the TOGA-switch on the MCP, or just auto-throttle with the TOGA-switch on the throttle levers. In addition, the execution of a go-around is a very procedural maneuver where pilots focus on a small number of specific aspects, such as thrust setting, establishing a positive rate of climb, gear and flap retraction, etc. Attention was not typically placed on aspects of secondary importance. Furthermore, only a few of the participants were successful at interpreting the updated safe envelope limits and understanding the reason for the stricter overspeed limit. Others were perplexed by the limits and one crew thought they were caused by a software error. This was an unintended consequence of the conscious decision not to brief specific aspects of each scenario in order to observe if the new limit displays were intuitive.

In spite of the factors contributing to the variability in the observed data, when the safe envelope boundaries were presented to the crews, most of them adapted their climb out profile. By reducing airspeed and climb rate, they maintained larger safety margins to the trim envelope boundaries and by doing so avoided loss of control over the aircraft pitching moment.

\section{Pilot Feedback}

From the answers in the pilot questionnaires, the following observations were made. The envelope limits increased awareness of events as well as aircraft energy. When rating the envelope information in order of usefulness, the crews preferred the airspeed limits above all others. The bank angle limits ranked second and the vertical speed limits were third. The vertical speed limits were also the smallest and newest aspect added to the PFD, and a few pilots commented to that effect. 


\section{Conclusion}

In past research, an online safe flight envelope estimation system was developed. Section $\amalg$ presented the approach, which combined rapid system identification, trim envelope estimation, and reachability analysis to determine sets of safe airspeeds and flight path angles from aerodynamic sensor information, current aircraft configuration, and the physics of flight dynamics. The outcome of the research was a source of information meant to enable safety assurance for control, automation, and crew systems.

Focusing on the crew system, the work presented in this paper developed an approach for transforming the safe flight envelope information into limit indicators for the PFD. The approach presented in section III leveraged current flightdeck indicator symbology to drive limit indicators for the airspeed and vertical speed tapes, which were further tied to new envelope driven bank and pitch limit indicators. The novel aspect of the approach was the method for driving the indicators from online envelope estimates, not the indicators themselves.

The integrated envelope estimation and limit displays were then implemented in a full motion simulator and tested by ten commercial crews flying relevant and challenging approach scenarios, as described in section [IV] The results discussed in section $\nabla$ demonstrated that for the icing scenario the new information provided by the envelope estimation technology was critical and enabled the crews to avoid low energy approaches, while for the unscheduled stabilizer scenario the new information helped pilots maintain safe higher energy operations. The pilots adapted their strategy based on the envelope information. In the icing scenario, flaps were deployed for higher speeds, which increased the safety margin to the boundaries of the trim envelope. In the stab trim scenario, the average climb during the go-around became shallower.

\section{Acknowledgments}

This work was supported by NASA's Aviation Safety Program, through the Vehicle Systems Safety Technologies (VSST) project, and by a Marie Curie International Outgoing Fellowship (IOF) within the 7th European Community Framework Program. The authors would like to acknowledge and sincerely thank Ramesh Panda, Ron Lehmer, Bogu Wei, Evelyn Hull and all others from the Crew Vehicle Simulator Research Facility (CVSRF) at NASA Ames Research Center, for their valuable contrabutions to the development of the simulation campaign through which this technology was tested.

\section{References}

\footnotetext{
1 "Statistical Summary of Commercial Jet Airplane Accidents - Worldwide Operations 1959 - 2011," Tech. rep., Boeing Commercial Aircraft, July 2012, Available at: http://www.boeing.com/news/techissues/pdf/statsum.pdf.

2 "CAST Safety Enhancement Plan," Technical Report SE207, Commercial Aviation Safety Team, 2013, Available at: www.skybrary.aero/index.php/Portal:CAST_SE_Plan.

${ }^{3}$ Tang, L., Roemer, M., Bharadwaj, S., and Belcastro, C., "An Integrated Health Assessment and Fault Contingency Management System for Aircraft," AIAA Guidance, Navigation and Control Conference, No. AIAA 2008-6505, August 2008.

${ }^{4}$ Goman, M., Khramtsovsky, A., and Kolesnikov, E., "Evaluation of Aircraft Performance and Maneuverability by Computation of Attainable Equilibrium Sets," Journal of Guidance, Control and Dynamics, Vol. 31, No. 2, March - April 2008, pp. 329-339.

${ }^{5}$ Keller, J. D., McKillip, R. M., and Kim, S., "Aircraft Flight Envelope Determination using Upset Detection and Physical Modeling Methods," AIAA Guidance, Navigation and Control Conference, No. AIAA 2009-6256, August 2009.

${ }^{6}$ Boskovic, J. D., Redding, J., and Knoebel, N., "An Adaptive Fault Management (AFM) System for Resilient Flight Control," AIAA Guidance, Navigation and Control Conference, No. AIAA 2009-6263, August 2009.

${ }^{7}$ Lombaerts, T., Schuet, S., Wheeler, K., Acosta, D., and Kaneshige, J., "Safe Maneuvering Envelope Estimation based on a Physical Approach," AIAA Guidance, Navigation and Control (GNC) Conference, No. AIAA-2013-4618, August 2013.

${ }^{8}$ Lombaerts, T., Schuet, S., Wheeler, K., Acosta, D., and Kaneshige, J., "Robust maneuvering envelope estimation based on reachability analysis in an optimal control formulation," Conference on Control and Fault-Tolerant Systems (SysTol), October 2013, pp. 318-323.

${ }^{9}$ Schuet, S., Lombaerts, T., Acosta, D., Wheeler, K., and Kaneshige, J., "An Adaptive Nonlinear Aircraft Maneuvering Envelope Estimation Approach for Online Applications," AIAA SciTech Guidance, Navigation and Control (GNC) Conference, No. AIAA 2014-0268, January 2014.
} 
${ }^{10}$ Kaneshige, J. and Gundy-Burlet, K., "Integrated neural flight and propulsion control system," AIAA Guidance, Navigation and Control Conference and Exhibit, No. AIAA-2001-4386, 2001.

11 "U.S. Standard Atmosphere," Tech. rep., Superintendent of Documents, October 1976.

${ }^{12}$ Lombaerts, T., Oort, E. V., Chu, Q., Mulder, J. A., and Joosten, D., "On-Line Aerodynamic Model Structure Selection and Parameter Estimation for Fault Tolerant Control," Journal of Guidance, Control and Dynamics, Vol. 33, No. 3, May-June 2010, pp. 707-723.

${ }^{13}$ Lombaerts, T., Fault Tolerant Flight Control - A Physical Model Approach, Phd dissertation, Delft University of Technology, May 2010, Available at: http://repository.tudelft.nl/view/ir/uuid\%3A538b0174-fe84-43af-954d-02f256b2ec50/.

${ }^{14}$ Lombaerts, T., Schuet, S., Acosta, D., and Kaneshige, J., "On-Line Flight Envelope Determination for Impaired Aircraft," submission to the EuroGNC Conference, April 2015.

${ }^{15}$ Lombaerts, T., Chu, Q., Mulder, J., and Joosten, D., "Real time damaged aircraft model identification for reconfiguring control," Proceedings of the AIAA AFM Conference and Exhibit, No. AIAA-2007-6717, August 2007.

${ }^{16}$ Lygeros, J., "On reachability and minimum cost optimal control," Automatica, Vol. 40, 2004, pp. 917-927.

${ }^{17}$ Govindarajan, N., An Optimal Control Approach for Estimating Aircraft Command Margin - with applications to Loss-Of-Control Prevention, Masters thesis, Delft University of Technology, October 2012, Available at: http://repository.tudelft.nl/view/ir/uuid\%3A48dbad61-728a-4c7e-ba3e-cf8382ef1cef/.

${ }^{18}$ Oort, E. V., Chu, P., and Mulder, J. A., Advances in Aerospace Guidance, Navigation and Control, chap. Maneuvering Envelope Determination through Reachability Analysis, Springer - Verlag, 2011, pp. 91-102.

${ }^{19}$ Kaneshige, J., Acosta, D., Schuet, S., Lombaerts, T., Shish, K., and Martin, L., "Trajectory Prediction and Alerting for Mode and Energy State Awareness," AIAA Scitech Infotech Conference, January 2015.

${ }^{20}$ Kaneshige, J., Benavides, J., Sharma, S., Martin, L., Panda, R., and Steglinski, M., "Implementation of a Trajectory Prediction Function for Trajectory Based Operations," AIAA Aviation Atmospheric Flight Mechanics Conference, No. AIAA 2014-2198, August 2014.

${ }^{21}$ Blake, M. W., "The NASA Advanced Concepts Flight Simulator - A unique transport aircraft research environment," AIAA Flight Simulation Technologies Conference, 1996. 